\title{
Detector of Optical Vortices as the Main Element of the System of Data Transfer: Principles of Operation, Numerical Model, and Influence of Noise and Atmospheric Turbulence
}

\author{
Valerii Aksenov, ${ }^{1}$ Igor Izmailov, ${ }^{2}$ Feodor Kanev, ${ }^{1}$ and Boris Poizner ${ }^{2}$ \\ ${ }^{1}$ Institute of Atmospheric Optics, Academician Zuev Square, Tomsk 634021, Russia \\ ${ }^{2}$ Tomsk State University, Tomsk, Russia \\ Correspondence should be addressed to Feodor Kanev, mna@iao.ru
}

Received 11 April 2011; Accepted 13 May 2011

Academic Editor: Paramasivam Senthilkumaran

Copyright (C) 2012 Valerii Aksenov et al. This is an open access article distributed under the Creative Commons Attribution License, which permits unrestricted use, distribution, and reproduction in any medium, provided the original work is properly cited.

The method is proposed of optical vortex topological charge detection along with a design of a corresponding detector. The developed technique is based on measurements of light field intensity. Mathematical model simulating performance of the detector is described in the paper, and results of numerical experiments are presented which illustrate recognition of a vortex in a turbulent medium and in the presence of amplitude and phase noise in the registered radiation. Influence of shifts of the system optical axis on precision of registration is also considered in the paper.

\section{Introduction}

As it is well known, optical beams not only can transmit energy, but also possess linear and angular momentum. The total angular momentum includes a spin component, associated with polarization of light, and orbital component, related with phase and amplitude profiles [1]. In the vicinity of a singular point, the complex amplitude of a beam carrying an optical vortex can be described by the equation [1]:

$$
A=C \cdot r^{V_{d}} \exp \left(j V_{d} \vartheta\right),
$$

where $C$ is a constant, $V_{d}$ is the topological charge of vortex, $\mathbf{r} \equiv(x, y)$ is the radius vector of a point in a cross-section of the beam, $r \equiv|\mathbf{r}|$ is the distance between the vortex and the given point, $\vartheta$ is an azimuth angle, and $j=\sqrt{-1}$ is an imaginary unit. The orbital angular momentum of such a beam reduced to one photon is equal to $\hbar V_{d}$. A topological charge $V_{d}$ can be associated with a bit of data. For example, $V_{d}=0$ can be considered as a logical zero, while $V_{d}=1$ is a logical unit. On the base of these properties the authors of $[2,3]$ proposed to use optical angular momentum to code and transfer information in optical communication systems.
Usually a laser beam propagating in a medium acquires some distortions, which reduce precision of data transfer. Intensity of distortions and probability of errors in communication line should be considered for each particular situation. But it is possible to presume that these errors depend on properties of the medium, on parameters of communication system, its design, principles of operation, and its practical realization. Obviously, influence of atmospheric turbulence and other factors on performance of the system using optical vortices to transfer information should be thoroughly considered. As an example of such analysis [4] can be taken where influence of distortions was discussed.

If construction of the communication system is chosen as the objective of investigation, structure of the system and influence of each element on its quality must be considered. Results of such investigation were published by the authors of [5]. The schematic of system discussed in this paper included transmitter, formed by generator of optical vortices, and initial wavefront corrector which includes adaptive receiver with a sensor of wavefront $S(\mathbf{r}, t)$, a second corrector, and comparator. The key element of the system is a sensor that registers topological charge $V_{d}(\mathbf{r}, t)$ of the vortex and its 
location $\mathbf{r}_{d}(t)$. Reconstruction of the wavefront is not always required in the algorithm of system performance; ordinarily the sensor is employed as a detector of vortices.

Such a detector can be built on the base of a unit measuring or reconstructing

(1) distributions of phase gradients (Shack-Hartmann sensor [6-8] or a pyramid wavefront sensor [9-11]),

(2) interference patterns (with subsequent analysis of it) $[12,13]$,

(3) distributions of white light intensity (with analysis of chromatic patterns) [14-16],

(4) phase distributions obtained out of local tilts measured by a wavefront sensor $[17,18]$.

But requirements to modern communication systems cannot be met with devices whose speed of response is less than a thousand Hz. In what follows we demonstrate the possibility to construct a detector of vortex topological charges using only measurements of beam intensity with registration speed of several $\mathrm{GHz}$.

\section{Detection of Optical Vortices with the Use of Interferometer}

In this part of the paper we illustrate theoretically the possibility to employ an interferometer as a detector of optical vortices in communication system. The obtained results show that existence and order of an optical vortex can be revealed with high precision even in the presence of additive phase noise with amplitude of $0.4 \pi$.

If a light field in a ring interferometer rotates on angle $\Delta=2 \pi M / m$, where the ratio of $M$ and $m$ is an even number, optical vortices of different topological charges included into the input field form different optical patterns in the output field. So the interferometer "sorts out" vortices into $m$ groups according to the rule [19]:

$$
\begin{gathered}
N_{s}=\left(m+\left[\left(V_{d}+L\right) \bmod m\right]\right) \bmod m, \\
\varphi_{\cos }=\varphi_{\cos 0}+\frac{2 \pi(L M)}{m},
\end{gathered}
$$

where $N_{s}$ is the number of a group (or a structure), expression "mod" signifies operation of a modulus calculation, $L$ is an even number, $\varphi_{\cos }$ is a phase shift in feedback contour, and $\varphi_{\cos 0}$ is a value of $\varphi_{\cos }$ corresponding to $L=0$ or $M=0$.

The described properties of the interferometer allow us to consider it as a detector of optical vortices, and especially suitable to this purpose is the Rozhdestvenskii interferometer with a unit of light field rotation included into an arm (unit $G)$ and with a phase shifting sell $(\varphi)$.

The input field $\mathbf{E}_{\mathrm{in}}(\mathbf{r}, t)$ of interferometer is divided into two parts. These parts propagate in different arms, experience different diffraction changes and different attenuation, and acquire different phase shifts. At the end of the interferometer optical paths, two fields interact. We assume that the attenuation of the fields in both arms is identical, as well as the lengths of the two optical paths. So the phase shift $\varphi$ between fields is induced only by unit $G$ and the phase shifting sell. This phase shift can be calculated as $\varphi=\omega \delta t$, where $\omega$ is the cycling frequency of the field and $\delta$ is the optical path difference induced by the sell.

Let's denote the optical field at the output of interferometer without the unit $G$ and the phase shifting sell as $\mathbf{E}(\mathbf{r}, t)$. Field $\mathbf{E}(\mathbf{r}, t)$ can be viewed as the output field of an "empty" interferometer with equal arms or as the input field $\mathbf{E}_{\mathrm{in}}(\mathbf{r}, t)$ recalculated to the output of such an interferometer. So without any limitations we can use field $\mathbf{E}(\mathbf{r}, t)$ instead of $\mathbf{E}_{\text {in }}(\mathbf{r}, t)$ and write the following equation for the output field:

$$
\mathbf{E}_{\Sigma}(\mathbf{r}, t)=\left(\frac{1}{2}\right)^{1 / 2}\left(\mathbf{E}(\mathbf{r}, t)+\mathbf{E}\left(\mathbf{r}^{\prime}, t-\delta t\right)\right) .
$$

Here $\mathbf{r}^{\prime} \equiv\left(x^{\prime}, y^{\prime}\right)$ is a point in the beam cross-section at the output of element $G$. Let's assume that

$$
\mathbf{E}(\mathbf{r}, t)=\mathbf{e}_{x} A(\mathbf{r}) \cos (\omega t+S(\mathbf{r}))
$$

where $A(\mathbf{r})$ and $S(\mathbf{r})$ are the amplitude and phase of lightfield and $\mathbf{e}_{x}$ is a unit vector parallel to axis $O x$. So the field at the output can be rewritten as

$$
\begin{aligned}
\mathbf{E}_{\Sigma}(\mathbf{r}, t)= & \mathbf{e}_{x}\left(\frac{1}{2}\right)^{1 / 2}[ \\
& +A(\mathbf{r}) \cos (\omega t+S(\mathbf{r})) \\
& \left.\left.+\mathbf{r}^{\prime}\right) \cos \left(\omega t+S\left(\mathbf{r}^{\prime}\right)-\varphi\right)\right] \\
\equiv & A_{\Sigma}(\mathbf{r}) \cos \left(\omega t+S_{\Sigma}(\mathbf{r})\right)
\end{aligned}
$$

Taking into account the formula for the beam intensity in free space $\left(\varepsilon_{0} / \mu_{0}\right)^{1 / 2}\left\langle\mathbf{E}^{2}(\mathbf{r}, t)\right\rangle_{T}\left(\varepsilon_{0}\right.$ is the electric constant and $\mu_{0}$ is the magnetic constant), we obtain the following equations describing the input and output fields:

$$
\begin{aligned}
I(\mathbf{r})= & 2\left\langle\mathbf{E}^{2}(\mathbf{r}, t)\right\rangle_{T}=A^{2}(\mathbf{r}), \\
I_{\Sigma}(\mathbf{r})= & 2\left\langle\mathbf{E}_{\Sigma}^{2}(\mathbf{r})\right\rangle_{T}=\frac{1}{2} I(\mathbf{r})+\frac{1}{2} I\left(\mathbf{r}^{\prime}\right) \\
& +\left(I(\mathbf{r}) I\left(\mathbf{r}^{\prime}\right)\right)^{1 / 2} \cos \left(S(\mathbf{r})-S\left(\mathbf{r}^{\prime}\right)+\varphi\right) \\
= & A_{\Sigma}^{2}(\mathbf{r}),
\end{aligned}
$$

where $\left\langle\mathbf{E}^{2}(\mathbf{r}, t)\right\rangle_{T} \equiv(1 / T) \int_{t}^{t+T} \mathbf{E}^{2}\left(\mathbf{r}, t^{\prime}\right) d t^{\prime}$, and coefficient $(1 / 2)\left(\varepsilon_{0} / \mu_{0}\right)^{1 / 2}$ was omitted to make the equation shorter. Output $I_{\Sigma} \equiv\left\langle I_{\Sigma}(\mathbf{r})\right\rangle_{\mathbf{r}}$ and input $I \equiv\langle I(\mathbf{r})\rangle_{\mathbf{r}}$ intensities averaged over the beam cross-section are

$$
\begin{aligned}
I & =\left\langle A^{2}(\mathbf{r})\right\rangle_{\mathbf{r}}, \\
I_{\Sigma} & =I+\left\langle\left[I(\mathbf{r}) I\left(\mathbf{r}^{\prime}\right)\right]^{1 / 2} \cos \left[S(\mathbf{r})-S\left(\mathbf{r}^{\prime}\right)+\varphi\right]\right\rangle_{\mathbf{r}} \\
& =\left\langle A_{\Sigma}^{2}(\mathbf{r})\right\rangle_{\mathbf{r}},
\end{aligned}
$$


$\langle I(\mathbf{r}, t)\rangle_{\mathbf{r}} \equiv\left(1 / S_{b}\right) \iint_{\left(S_{b}\right)} I(\mathbf{r}, t) d \mathbf{r}$, and $\left(S_{b}\right)$ is the region of square $S$, occupied by the beam.

The relative intensity of the output beam is possible to introduce in the following way:

$$
\begin{aligned}
I_{r} \equiv & \frac{I_{\Sigma}}{I}=1+I^{-1} \\
& \cdot\left\langle\left[I(\mathbf{r}) I\left(\mathbf{r}^{\prime}\right)\right]^{1 / 2} \cos \left[S(\mathbf{r})-S\left(\mathbf{r}^{\prime}\right)+\varphi\right]\right\rangle_{\mathbf{r}} .
\end{aligned}
$$

Obviously, this intensity is equal to the ratio of the output and input power of the beam; therefore, $I_{r}$ can be registered experimentally.

Let's assume that the vortex light field incident on the input aperture of interferometer includes only one singular point positioned in the origin of coordinate system. In general, amplitude $A(\mathbf{r})$ and phase $S(\mathbf{r})$ profiles of the field entering (4) are the following:

$$
A(\mathbf{r})=A(r), \quad S(\mathbf{r})=S_{r}(r)+V_{d} \cdot \vartheta(\mathbf{r}),
$$

and $\vartheta(\mathbf{r}) \equiv \arg (\mathbf{r})$ is an azimuth angle, while $V_{d}$ is the order of a singular point. In more strict representation these profiles can be written as

$$
A(\mathbf{r})=C \exp \left(-\frac{r^{2}}{r_{0}^{2}}\right)\left(\frac{r}{\rho V}\right)^{\left|V_{d}\right|}, \quad S(\mathbf{r})=V_{d} \cdot \vartheta(\mathbf{r})
$$

Here $r_{0}$ is the radius of a Gaussian beam with zero dislocation $\left(V_{d}=0\right)$ and $\rho V$ is a coefficient defining the dependence of the beam amplitude on radial coordinate. Vectors $\mathbf{r}$ and $\mathbf{r}^{\prime}$ are related by rotational transformation; modules of these vectors are equal $\left(r=r^{\prime}\right)$, and $\vartheta(\mathbf{r})=\vartheta\left(\mathbf{r}^{\prime}\right)+M \Delta$, so substitution of (9) into (8) gives us the relative intensity

$$
I_{r}\left(V_{d}\right) \equiv \frac{I_{\Sigma}}{I}=1+\cos \left[\left(\frac{2 \pi M V_{d}}{m}\right)+\varphi\right] .
$$

Several important properties of a beam with intensity defined by (11) are recorded below.

(1) Variations of topological charge $V_{d}$ produce a discrete series of intensity $I_{r}$ values, $I_{r}\left(V_{d}\right)=I_{r}\left(V_{d}+i m\right)$, where $i$ is an arbitrary even number.

(2) Influence of a vortex on intensity $I_{r}$ can be compensated by appropriate variations of phase shift $\varphi$. In the same way as it was done in (2), the phase shift is possible to be represented as $\varphi_{0}+2 \pi(L M) / m$, where $L, M$, and $m$ are even numbers and $\varphi_{0}$ is a phase shift corresponding to $L=0$.

(3) Magnitude of $I_{r}$ does not depend on amplitude $A(r)$ and phase distribution $S_{r}(r)$ entering (4). Moreover, (11) is valid for a general case of rotation symmetry of $m$ th order (in the optical system of interferometer, rotation is induced by element $G)$. According to this property, $A(\mathbf{r})=A\left(\mathbf{r}^{\prime}\right)$ and $S(\mathbf{r})-S\left(\mathbf{r}^{\prime}\right)=$ const + $2 \pi i$, that is, amplitude and phase profiles are identical in different regions of cross-section cut by rays with azimuth angles $\vartheta=\Delta i$.
The first and second properties strictly follow from (2). Numeration of structures is the same as earlier, but it can also be viewed as the rule of $I_{r}$ discretization according to which number $N_{s}$ corresponds to structure $I_{r N s}$.

Obviously, if a field with the symmetry discussed in Section 3 is generated by a remote source, its properties do not change in vacuum; consequently, the distance from a source to detector is not important.

More general assumption stating that intensity $I_{r}$ does not depend on the distance between the source and detector can be proved for beams propagating in vacuum or in linear homogeneous medium.

Really, diffraction of a beam in such a medium is described mathematically by a linear operator $L_{D}$. Transformations of a beam in the detector (splitting operation of the beam into three parts $\left(L_{\mathrm{Spl}}\right)$, rotation of one part by element $G\left(L_{G}\right)$, and summation of two parts $\left.\left(L_{\Sigma}\right)\right)$ are also linear. Let's rearrange these operators. Due to linearity of operator $L_{D}$, the results obtained as the sum of the fields after diffraction is the same as results of diffraction of the summed fields. Because of that, mirrors $M_{1}$ and $M_{4}$ can be shifted to the source of radiation, that is, $L_{\mathrm{Spl}} L_{D} \mathbf{E} \equiv L_{D} \mathbf{E}_{1}+L_{D} \mathbf{E}_{2}+L_{D} \mathbf{E}_{3}=$ $L_{D}\left(\mathbf{E}_{1}+\mathbf{E}_{2}+\mathbf{E}_{3}\right) \equiv L_{D} L_{\mathrm{Spl}} \mathbf{E}$. The order of application of operators $L_{D}$ and $L_{G}$ can also be changed: $L_{G} L_{D} \mathbf{E}=L_{D} L_{G} \mathbf{E}$, because diffraction of the field and its subsequent rotation gives the same result as rotation and diffraction of the field. Physically, this means that element $G$ can be moved into the plane of the field generator. Using again the linearity of operator $L_{D}$, we came to a conclusion about the possibility of changing the order of operators $L_{D}$ and $L_{\Sigma}: L_{\Sigma}\left(L_{D} \mathbf{E}_{1}, L_{D} \mathbf{E}_{2}\right)=L_{D} \mathbf{E}_{1}+L_{D} \mathbf{E}_{2}=L_{D}\left(\mathbf{E}_{1}+\mathbf{E}_{2}\right)=$ $L_{D} L_{\Sigma}\left(\mathbf{E}_{1}, \mathbf{E}_{2}\right)$. So mirrors $M_{2}$ and $M_{3}$ are also possible to move to the source of radiation.

As a result of all these operations, the interferometer is shifted to the source of radiation while detectors of intensity registering the fields $\mathbf{E}_{\Sigma}$ and $p \mathbf{E}$ remain near the receiver. Let's direct two beams of light from the source to the receiver. One of them (field $\mathbf{E}_{\Sigma}$ ) is taken from the output of interferometer, and the other $(p \mathbf{E})$ is reflected by mirror $M_{4}$. Obviously, the ratio of intensity of these two beams does not depend on the length of paths passed by them. In other words, value of $I_{r} \equiv$ $I_{\Sigma} / I$ is independent of the path length.

In analysis of the problem we should take into account aberrations of beams that are always present in real systems. To do so we insert into the phase of field described by (9) white additive noise and into its amplitude white multiplicative noise with amplitudes $A_{n S}$ and $A_{n A}$ correspondingly:

$$
\begin{gathered}
A(\mathbf{r})=A(r)\left(1+A_{n A} \xi_{A}(\mathbf{r})\right), \\
S(\mathbf{r})=S_{r}(r)+V_{d} \cdot \vartheta(\mathbf{r})+A_{n S} \xi_{S}(\mathbf{r}),
\end{gathered}
$$

where $\xi_{A}(\mathbf{r}) \in[-1,1]$ and $\xi_{S}(\mathbf{r}) \in[-1,1]$ are random functions of $\mathbf{r}$ uniformly distributed in interval $[-1,1]$. This functions should meet the requirement

$$
\begin{aligned}
\left\langle\xi_{A}(\mathbf{r})\right\rangle_{\mathbf{r}} & =0, & & \left\langle\xi_{S}(\mathbf{r})\right\rangle_{\mathbf{r}}=0, \\
\left\langle\xi_{A}(\mathbf{r}) \xi_{A}\left(\mathbf{r}^{\prime}(\mathbf{r})\right)\right\rangle_{\mathbf{r}} & =0, & & \left\langle\xi_{S}(\mathbf{r}) \xi_{S}\left(\mathbf{r}^{\prime}(\mathbf{r})\right)\right\rangle_{\mathbf{r}}=0
\end{aligned}
$$


if $\mathbf{r}^{\prime}(\mathbf{r}) \neq \mathbf{r}$. Mathematically these conditions signify that spatial scale of noise variations is much smaller than the characteristic size of our problem, for example, lesser than the length of a ring $2 \pi r_{c} / m$ of radius $r_{c}$. Substituting (12) into (8), we obtain for intensity $I_{r}$ the following formula:

$$
\begin{aligned}
I_{r}=1+I^{-1}\langle & A(\mathbf{r})^{2}\left(1+A_{n A} \xi_{A}(\mathbf{r})\right)\left(1+A_{n A} \xi_{A}\left(\mathbf{r}^{\prime}\right)\right) \\
& \left.\times \cos \left[\frac{2 \pi V_{d} M}{m}+\varphi_{0}+A_{n S}\left(\xi_{S}(\mathbf{r})-\xi_{S}\left(\mathbf{r}^{\prime}\right)\right)\right]\right\rangle_{\mathbf{r}} .
\end{aligned}
$$

In what follows we consider two specific cases: $A_{n A} \neq 0$, $A_{n S}=0$ and $A_{n A}=0, A_{n S} \neq 0$. For the first situation, (14) can be written as

$$
\begin{aligned}
I_{r}= & +I^{-1}\left\langle A(\mathbf{r})^{2}\left(1+A_{n A} \xi_{A}(\mathbf{r})\right)\left(1+A_{n A} \xi_{A}\left(\mathbf{r}^{\prime}\right)\right)\right\rangle_{\mathbf{r}} \\
& \times \cos \left(\frac{2 \pi V_{d} M}{m}+\varphi\right) .
\end{aligned}
$$

This equation is possible to simplify with application of several mathematical operations:

(1) transmission from Cartesian to polar coordinates in procedure of averaging \langle\rangle$_{\mathbf{r}}=\left\langle\langle r\rangle_{\vartheta}\right\rangle_{r}=$ $\left(1 / S_{b}\right) \int_{0}^{r_{\max }} \int_{0}^{2 \pi} r d r d \vartheta$

(2) calculation of integral over angle $\vartheta$ using such properties of average values as $\left\langle\xi_{A}(\mathbf{r})\right\rangle_{\mathbf{r}}=0$ and $\left\langle\xi_{A}(\mathbf{r}) \xi_{A}\left(\mathbf{r}^{\prime}(\mathbf{r})\right)\right\rangle_{\mathbf{r}}=0$ for $\mathbf{r}^{\prime}(\mathbf{r}) \neq \mathbf{r}$ which are valid in any cross-section of the beam. When calculation of this integral is impossible, we can change averaging over angle $\vartheta$ (this angle is analogous to some extent with a temporal variable) to averaging over the ensemble of realizations. For example,

$$
\left\langle\xi_{A}(\mathbf{r})^{2}\right\rangle_{\vartheta}=\int_{-\infty}^{\infty} \rho\left\{\xi_{A}(\mathbf{r})\right\} \xi_{A}(\mathbf{r})^{2} d \xi_{A}(\mathbf{r}),
$$

where the density of probability of random process $\xi_{A}(\mathbf{r})$ is $\rho\left\{\xi_{A}(\mathbf{r})\right\}=1 / 2$ for $\xi_{A}(\mathbf{r}) \in[-1,1]$ and $\rho\left\{\xi_{A}(\mathbf{r})\right\}=0$ for $\xi_{A}(\mathbf{r}) \notin[-1,1]$,

(3) returning to the Cartesian coordinates, for example,

$$
\begin{aligned}
\left\langle r A(r)^{2}\right\rangle_{r} & =\left\langle r A(r)^{2}\langle 1\rangle_{\vartheta}\right\rangle_{r}=\left\langle r\left\langle A(r)^{2}\right\rangle_{\vartheta}\right\rangle_{r} \\
& =\left\langle A(r)^{2}\right\rangle_{r}=I .
\end{aligned}
$$

In such a way, from (15), we obtain a short formula

$$
I_{r}=1+\left(1+\frac{A_{n A}^{2}}{3}\right)^{-1} \cdot \cos \left(\frac{2 \pi V_{d} M}{m}+\varphi\right)
$$

Amplitude of noise $A_{n A}$ should always be less than unity, so with $A_{n A}=1$ we obtain the minimum value of coefficient before cosign function in (18). This coefficient is equal to $(1+1 / 3)^{-1}=0.75$. The value of coefficient is close to unity, which means that influence of multiplicative amplitude noise on intensity $I_{r}$ is rather small (but only in absence of phase noise), so value $V_{d}$ of topological charge is possible to detect correctly in the presence of such noise. This property will be illustrated later by results of numerical experiments.

Assuming that $A_{n A}=0$ and $A_{n S} \neq 0$, let's analyze the second problem concerning the influence of noise on the quality of vortex registration. In this assumption (14) can be rewritten in the form

$$
\begin{aligned}
I_{r}= & 1+I^{-1} \\
& \cdot\left\langle A(r)^{2} \cos \left[\frac{2 \pi V_{d} M}{m}+\varphi+A_{n S}\left(\xi_{S}(\mathbf{r})-\xi_{S}\left(\mathbf{r}^{\prime}\right)\right)\right]\right\rangle_{\mathbf{r}}
\end{aligned}
$$

Viewing this equation as a function of two independent random arguments and applying the operations discussed earlier, we obtain

$$
I_{r}=1+\left(\frac{\sin ^{2}\left(A_{n S}\right)}{A_{n S}^{2}}\right) \cos \left(\frac{2 \pi V_{d} M}{m}+\varphi\right)
$$

According to this formula the phase noise decreases the difference between values of $I_{r}$ registered for different topological charges as $\sin ^{2}\left(A_{n S}\right) / A_{n S}^{2}$ making detection of the charge more difficult. For example, if $A_{n S}=\pi$, detection of a vortex is absolutely impossible.

The assumptions made above allow us to write for $I_{r}$ a more general equation:

$$
I_{r}=1+\left(1+\frac{A_{n S}^{2}}{3}\right)^{-1}\left(\frac{\sin ^{2}\left(A_{n S}\right)}{A_{n S}^{2}}\right) \cos \left(\frac{2 \pi V_{d} M}{m}+\varphi\right) .
$$

\section{Simulation of the Vortex Detector Performance and Analysis of Its Characteristics in the Presence of White Amplitude and Phase Noise}

To validate conclusions made in the previous paragraph, especially properties of (21), we developed the numerical model of the detector which simulates performance of the device in the presence of additive phase noise and multiplicative amplitude noise with amplitudes $A_{n S}$ and $A_{n A}$. This noise is superimposed on the field described by (10) with parameter $\rho V=8 r_{0}$. In the model of detector, the field $\mathbf{E}_{\Sigma}(\mathbf{r}, t)=(1 / 2)^{1 / 2}\left(\mathbf{E}(\mathbf{r}, t)+\mathbf{E}\left(\mathbf{r}^{\prime}, t-\delta t\right)\right)$ is calculated along with intensities $I_{\Sigma} \equiv\left\langle I_{\Sigma}(\mathbf{r}, t)\right\rangle_{\mathbf{r}}, I \equiv\langle I(\mathbf{r}, t)\rangle_{\mathbf{r}}$, and $I_{r} \equiv I_{\Sigma} / I$. Parameter $M$ defining the angle of rotation was taken equal unity $(M=1)$, and the path length was equal zero.

The density of probability obtained in 150 realizations for relative intensity $I_{r}$ is shown in Figures 2 and 3 for several fixed values of $A_{n S}, A_{n A}$, and $V_{d}$. Results of numeric experiments illustrated in Figures 2 and 3 were obtained with different angles of rotation $\Delta$ and different phase shifts $\varphi$. The magnitude of phase shift $\varphi$ was specially chosen to provide maximum difference in values of intensity $I_{r}\left(V_{d}\right)$. 


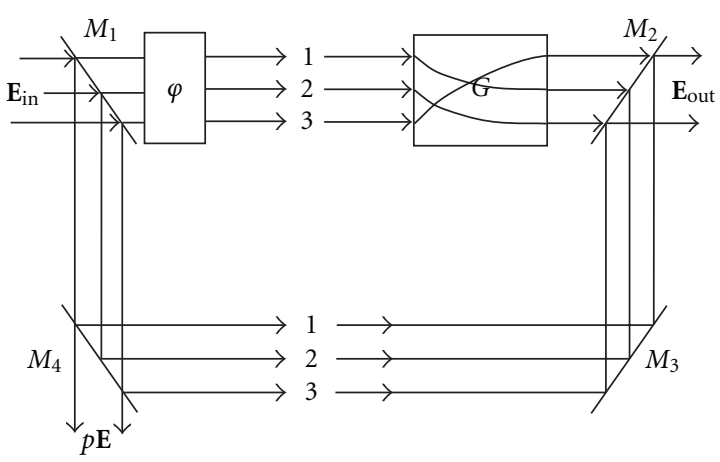

Figure 1: The modified Rozhdestvenskii interferometer as a detector of optical vortices. Rotation of rays 1, 2, and 3 is shown for the angle of $120^{\circ}$.

For example, $\varphi=0$ for $\Delta=180^{\circ}$, and $\varphi=-90^{\circ}$ for $\Delta=120^{\circ}$.

The main properties of the detector can be systematized as follows.

(1) Performance of the detector is influenced mainly by phase noise; influence of amplitude noise is insignificant. This thesis coincides with conclusions obtained in analysis of (18) and (20).

(2) Detection of a vortex topological charge $V_{d}$ is possible in a single realization with applications of the following rules:

$V_{d}=\left\{\begin{array}{ll}1+2 i, & I_{r} \leq 1, \\ 0+2 i, & I_{r}>1,\end{array} \quad\right.$ for $\Delta=180^{\circ}$,

$V_{d}=\left\{\begin{array}{ll}2+3 i, & I_{r}<0.94, \\ 0+3 i, & I_{r} \in[0.94,1.06] \\ 1+3 i, & I_{r}>1.06,\end{array}\right.$ for $\Delta=120^{\circ}$,

where $i$ is an arbitrary even number. These rules are valid with $A_{n S} \leq 0.64 \pi$ (for $\Delta=120^{\circ}$ ) and with $A_{n S} \leq 0.72 \pi$ (for $\Delta=180^{\circ}$ ). The borders of their application are shown in Figures 2 and 3 by vertical dashed lines.

(3) The averaging of results over large numbers of realizations allows one to detect value of topological charge $V_{d}$ even with $A_{n S} \leq 0.85 \pi$ (for angle $\Delta=180^{\circ}$ as well as for $\left.\Delta=120^{\circ}\right)$. But in this case the value of mean intensity $\left\langle I_{r}\right\rangle$ should be used instead of $I_{r}$. This result is not converse with conclusions made in analysis of (20) where $A_{n S}<\pi$.

In Figure 2 as well as in Figure 3 distributions of intensity $I_{r}$ have finite width, and due to this fact curves corresponding to maximum and minimum values of $I_{r}$ in Figure 4 do not coincide. This can be attributed to small resolution of calculation grid with only $32 \times 32$ nodes in planes transverse relatively to the direction of propagation. So conditions $\left(\left\langle\xi_{A}(\mathbf{r})\right\rangle_{\mathbf{r}}=0,\langle\xi S(\mathbf{r})\rangle_{\mathbf{r}}=0,\left\langle\xi_{A}(\mathbf{r}) \xi_{A}\left(\mathbf{r}^{\prime}(\mathbf{r})\right)\right\rangle_{\mathbf{r}}=0\right.$, and $\left\langle\xi_{S}(\mathbf{r}) \xi_{S}\left(\mathbf{r}^{\prime}(\mathbf{r})\right)\right\rangle_{\mathbf{r}}=0$ with $\mathbf{r}^{\prime}(\mathbf{r}) \neq r$, ) used to obtain (18),
(20), and (21) are met only approximately. This inaccuracy can be removed completely in the process of averaging; for instance, curves representing $\left\langle I_{r}\right\rangle_{N r}$ coincide with values of $I_{r}$ calculated along (21).

In the end of the paragraph we want to point out that theoretical conclusions concerning influence of white noise on performance of detector are supported by results of numerical experiments.

\section{Influence of Optical Axis Shifts on the Value of Relative Intensity $I_{r}$}

As the origin of white noise, such imperfections of optical system can be taken as roughness of optical surfaces of detector and generator and some other factors. Another source of imperfections is relative shift $S_{h x}$ of optical axes of detector and generator.

To assess the influence of $S_{h x}$ on $I_{r}$, we have simulated field transformations in the detector. In contrast to the situation described in the previous paragraph we assumed that aberrations of field were absent. As earlier, we took $\varphi=0$ for $\Delta=180^{\circ}$ and $\varphi=-90^{\circ}$ for $\Delta=120^{\circ}$ (Figure 1). The obtained dependencies of $I_{r}$ on $S_{h x} / r_{0}$ are shown in Figure 5 for different parameters of the problem.

In the numerical model, the field described by (10) can be used as distribution of radiation at the output of generator and, at the same time, as the field at the output of interferometer. This approach is valid because intensity $I_{r}$ does not depend on the path length. So as a factor of normalization we used the size $r_{0}$ of the beam at the output of the laser source.

The following characteristic features of the problem are seen in Figure 5. In situations where the finite size of the receiver aperture does not influence the results, relative shifts of axes induce asymptotical approach of $I_{r}$ to unity. Also we observe oscillations of $I_{r}$ which can be registered for topological charges different from zero $\left(V_{d} \neq 0\right)$.

In experiments performed with angle of rotation $\Delta=$ $120^{\circ}$, the finite size of aperture manifests itself, and with the increase of the ratio $S_{h x} / r_{0}$ intensity, $I_{r}$ approaches zero. This can be attributed to some properties of numerical model, namely, to the fact that due to rotation of the filed some part of interferometer input signal does not fall into the limits of the output aperture.

Oscillations of $I_{r}$ induce intersections of dependences $I_{r}\left(S_{h x} / r_{0}\right)$ obtained for different charges $V_{d}$ (it should be noted that for different charges values of $I_{r}(0)$ are different). This means that for some shifts of axis, detection of topological charge with rules given by (22) is impossible.

Frequency and amplitude of oscillation increase with the increase of absolute values of topological charge. So for large $\left|V_{d}\right|$ intersections, points are observed for less relative shifts $S_{h x} / r_{0}$. For example, if angle $\Delta$ equals 180 degrees $\left(\Delta=180^{\circ}\right)$, intersection of curves corresponding to the pair $\left(V_{d 0}, V_{d 1}\right)=$ $(1,2)$ happens earlier than for pair $(3,2)$ but later than for $\left(V_{d 0}, V_{d 1}\right)=(3,0)$.

The presence of coefficient $(r / \rho V)^{\left|V_{d}\right|}$ in (10) results in an increase of amplitude of oscillations and in a decrease of their 


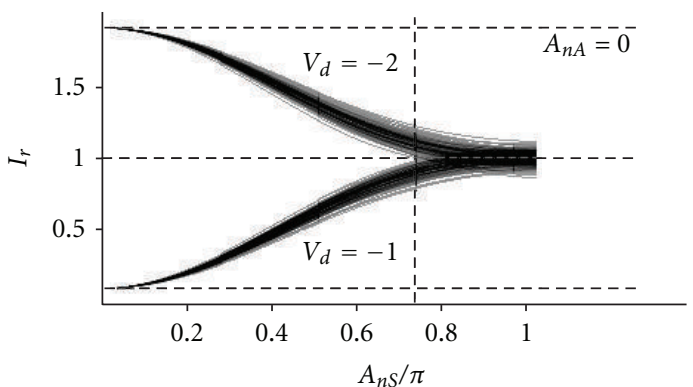

(a)

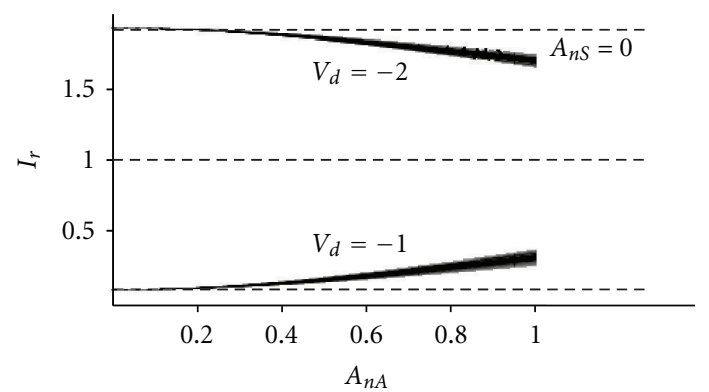

(c)

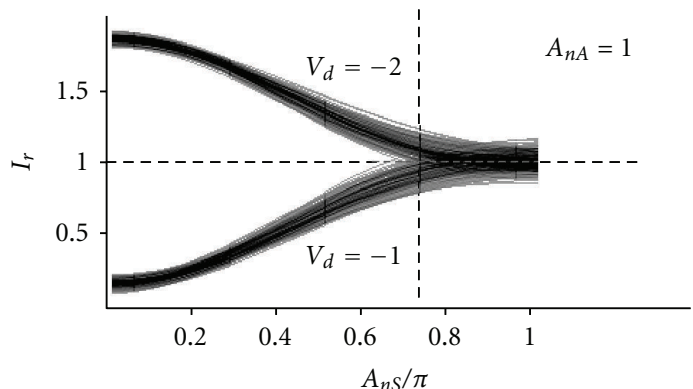

(b)

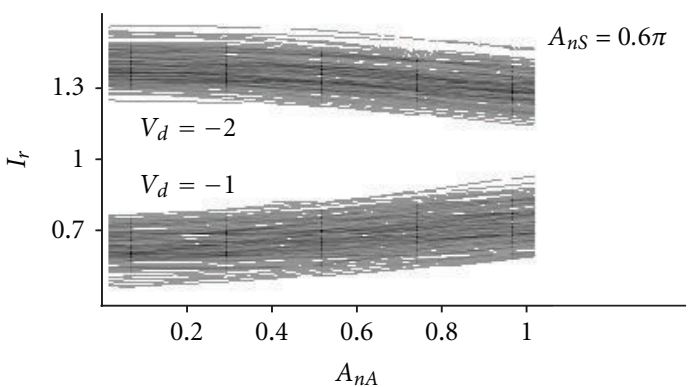

(d)

Figure 2: Influence of phase and amplitude noise with amplitudes $A_{n S}$ and $A_{n A}$ on the probability density of the intensity $I_{r}$ with $\Delta=180^{\circ}$ and $\varphi=0$. The vertical dashed line corresponds to $A_{n S}=0.72 \pi$, while horizontal dashed lines correspond to relative intensity $I_{r}$ equal to 0 , 1 , and 2 .

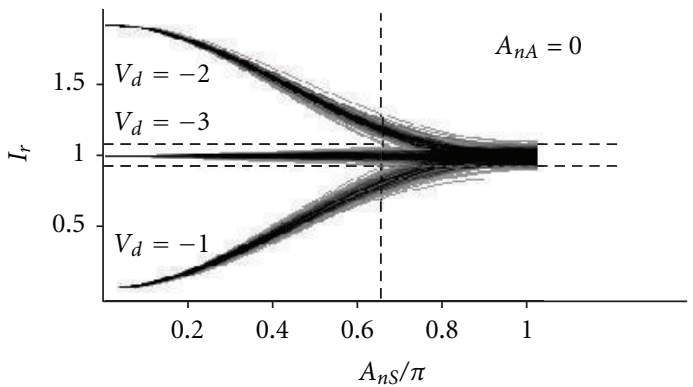

(a)

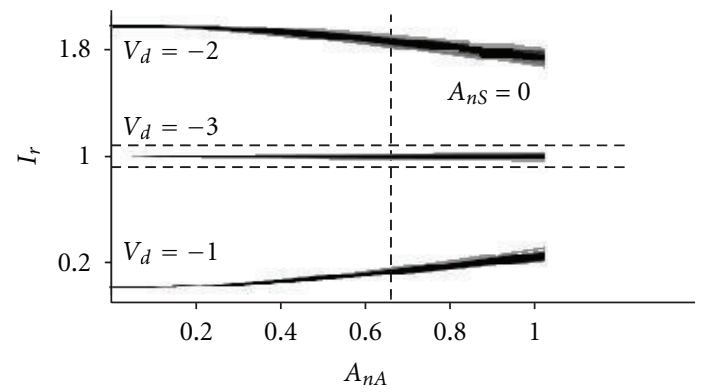

(c)

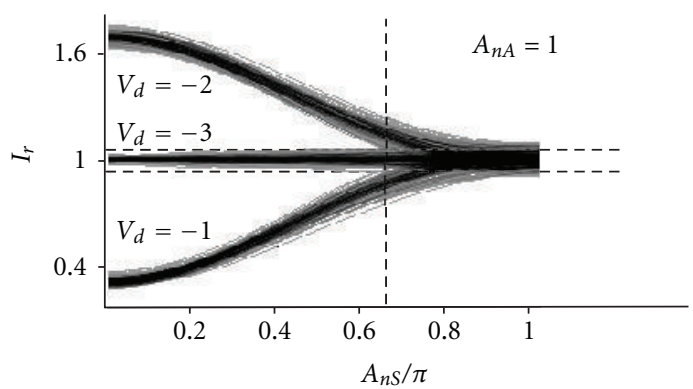

(b)

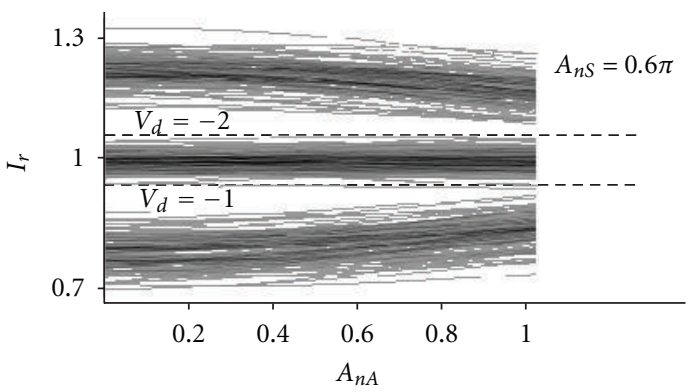

(d)

Figure 3: The same results as in Figure 2 obtained with $\Delta=120^{\circ}$ and $\varphi=\pi / 2$. The vertical dashed line corresponds to $A_{n s}=0.64 \pi$, while horizontal dashed lines correspond to relative intensity $I_{r}$ equal to 0.94 and to 1.06 . 


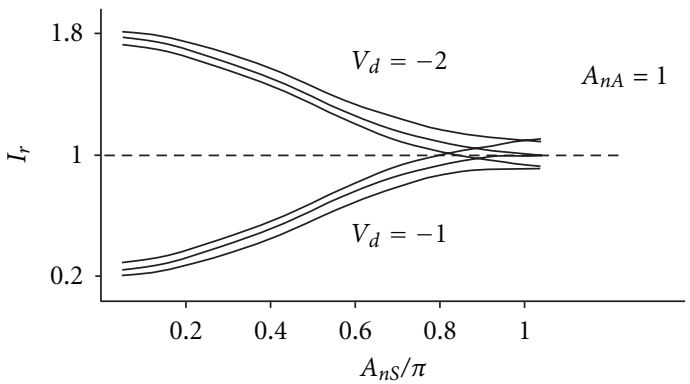

(a)

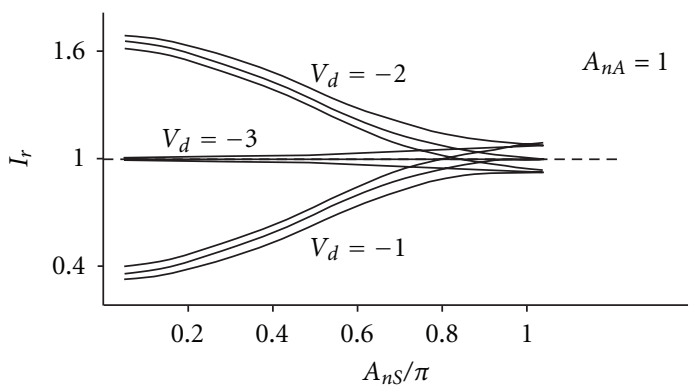

(c)

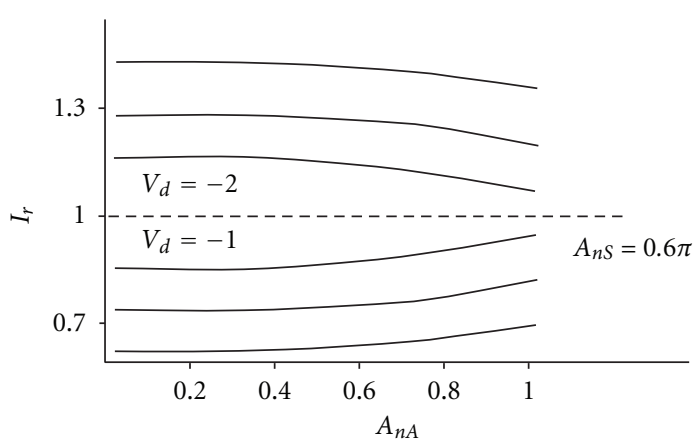

(b)

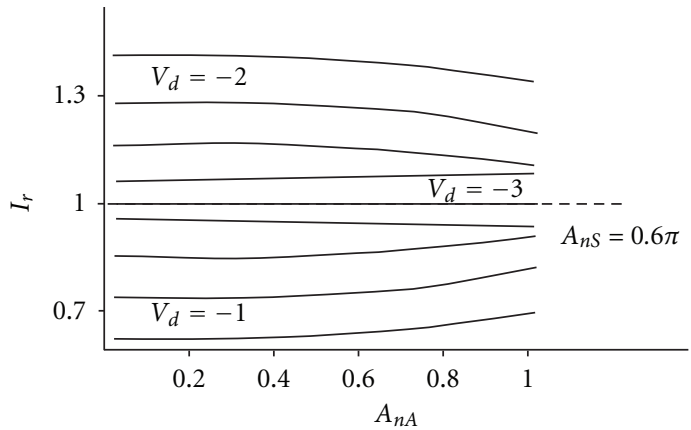

(d)

Figure 4: Influence of phase and amplitude noise with amplitudes $A_{n S}$ and $A_{n A}$ on maximum and mean values of intensity $\left\langle I_{r}\right\rangle$ calculated over 150 realizations. Results were obtained with $\Delta=180^{\circ}$ and $\varphi=0(\mathrm{a}, \mathrm{b})$ and with $\Delta=180^{\circ}$ and $\varphi=0$ (c, d). Amplitudes of noise were taken as $A_{n A}=0.5(\mathrm{a}, \mathrm{c})$ and $A_{n S}=0.6 \pi(\mathrm{b}, \mathrm{d})$. The mean values of intensity calculated according to (21) coincide with curves in figures.
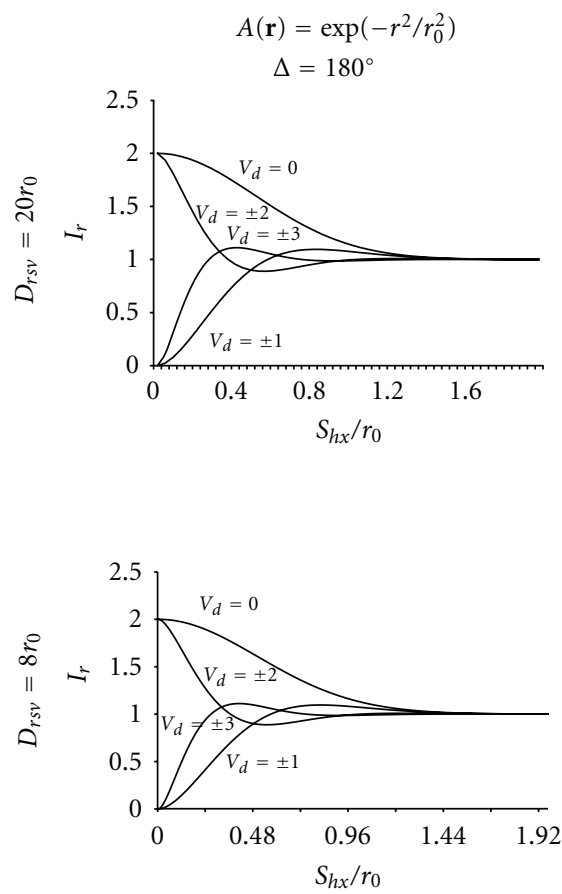

$$
\begin{gathered}
A(\mathbf{r})=\exp \left(-r^{2} / r_{0}^{2}\right) \\
\Delta=120^{\circ}
\end{gathered}
$$

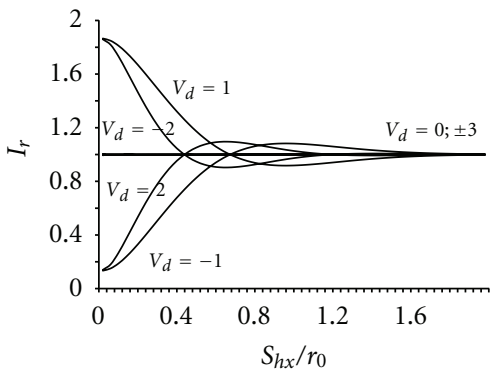

(a)

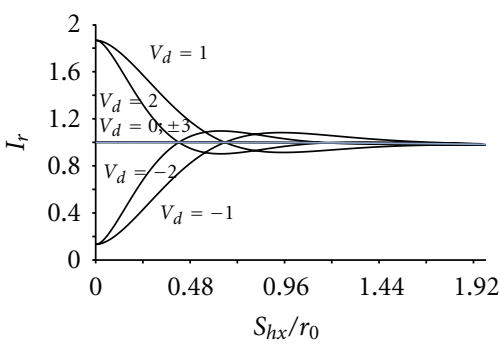

(b)
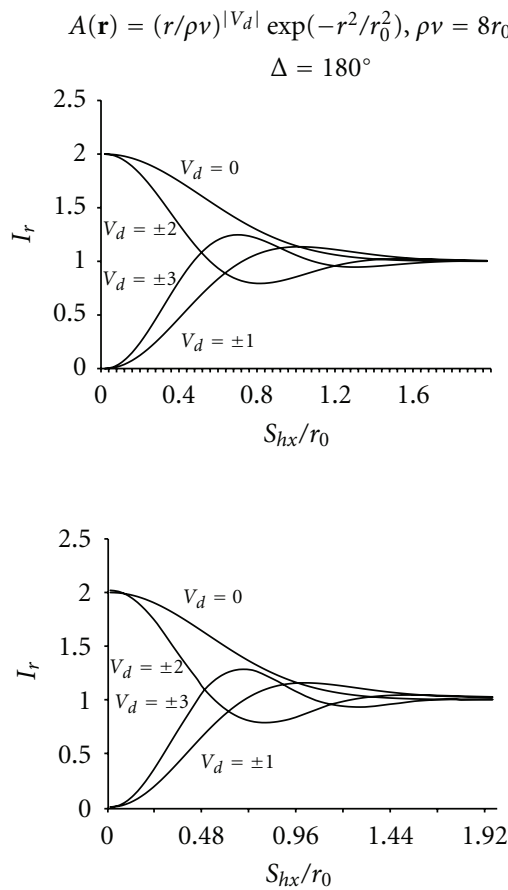

FIGURE 5: Dependence of relative intensity of interference field $I_{r}\left(S_{h x} / r_{0}\right)$ on the shift of optical axes $S_{h x}$ obtained for different initial amplitude profiles, angles of rotation $\Delta$, and topological charges $V_{d}$. 


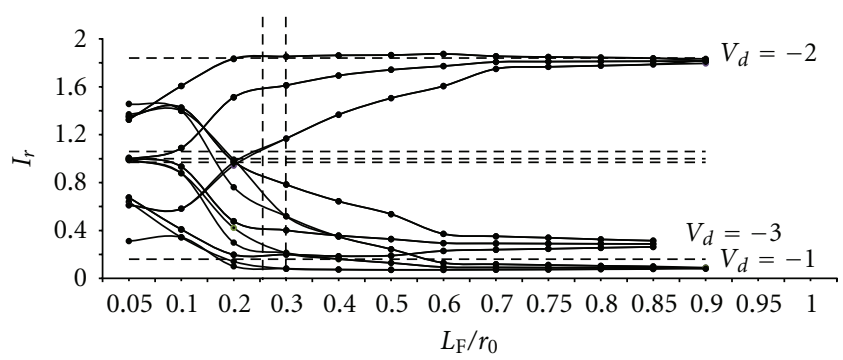

(a)

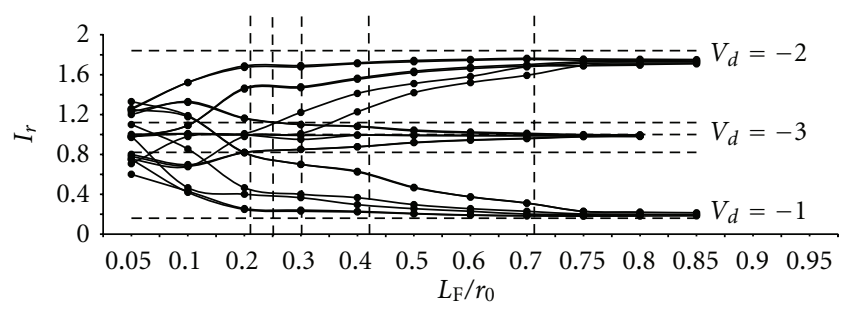

(b)

Figure 6: Calculated over 100 realizations, mean and maximum values of $I_{r}$ obtained with different topological charges $V_{d}$ for angles of rotation $\Delta=180^{\circ}$ (a) and $\Delta=120^{\circ}$ (b). Here $L_{\mathrm{F}}$ is Fried's coherence length. Horizontal dashed lines in the upper and lower parts of the pictures show the regions where values of $I_{r}$ are greater and lesser than 1.84 and 0.16 . In the middle part of Figure 5(a) dashed lines correspond to $I_{r}=1$ with threshold values $I_{r}=0.97$ and $I_{r}=1.06$; in Figure 5(b) they correspond to $I_{r}=1$ with threshold values $I_{r}=0.82$ and $I_{r}=1.12$. Vertical dashed lines in Figure 5(a) show values of $L_{\mathrm{F}} / r_{0 B}$ equal to 0.26 and 0.30 , while in Figure 5 (b) equal to $0.22,0.25,0.30,0.43$, and 0.71 .

frequency. For topological charges with modulus greater than zero, this coefficient shifts points of intersection along $S_{h x} / r_{0}$ axes on $25-56 \%$, approximately from $S_{h x}=0,5 r_{0}$ up to $S_{h x}=0,7 r_{0}$. At the same time, the curve corresponding to $V_{d}=0$ does not change its position, so the difference between curves becomes greater. This feature is especially well pronounced for the pair of charges $\left(V_{d 0}, V_{d 1}\right)=( \pm 3,0)$.

The intersections discussed above are observed to be of relatively small values of $S_{h x}\left(S_{h x} \approx 0.3 r_{0}, \ldots, 0.8 r_{0}\right)$. So it is not necessary to register the whole distribution of the beam amplitude by the receiver. Really, for $S_{h x} \in\left[0,0.8 r_{0}\right]$, the curve representing dependence $I_{r}\left(S_{h x} / r_{0}\right)$ for the diameter of aperture $D_{\mathrm{rsv}}=20 r_{0}$ coincides with the curve obtained with the aperture $D_{\text {rsv }}=4 r_{0}$. Moreover, for $S_{h x}=0$, intensity $I_{r}$ is practically not influenced by the aperture diameter $D_{\text {rsv }}$.

\section{Detection of a Vortex Carried by a Beam in the Turbulent Atmosphere}

White noise is a source of aberrations that can easily be simulated in numerical experiments, but distortions of beams in the real atmosphere cannot be related explicitly only with noise, so we assessed the influence of turbulence on performance of the detector. Investigations were carried out for beams with Gaussian initial distribution of amplitude and with a screw dislocation in the wavefront. The beam formed in such a way passed a thin phase screen and an optically homogeneous path of length $L_{t}$.

Intensity of turbulent distortions was characterized by the Fried coherence length $L_{F}$ and by inner $M_{\text {inner }}$ and outer $M_{\text {outer }}$ scales of turbulence. Value of $L_{F}$ was varied from $50 r_{0 B}$ to $0.05 r_{0 B}$, inner and outer scales: $M_{\text {inner }}=0.16 r_{0 B} \approx$ $0.11 r_{0}, M_{\text {outer }}=5 r_{0 B} \approx 3.5 r_{0}$, or $M_{\text {outer }}=20 r_{0 B} \approx 14.14 r_{0}$, where $r_{0 \mathrm{~B}}=r_{0} / \sqrt{2}$ is a radius of a Gaussian beam entering the equation. $I(\mathbf{r})=C^{2} \exp \left(-2 r^{2} / r_{0}^{2}\right)=C^{2} \exp \left(-r^{2} / r_{0 B}^{2}\right)$. Path length was taken equal to 0.05 and 0.5 of diffraction length that corresponds to 5.9 and 59 kilometers for beams with radius of $10 \mathrm{~cm}$ and wavelength of $0.5 \mathrm{mkm}$. Averaged over 100 realizations of random phase screen, the minimum, maximum, and mean values of intensity $I_{r}$ were calculated for all combinations of the physical parameters listed above.

On the path of 59 kilometers in the registration plane the distance between nodes of calculation grid was about $r_{0 B}{ }^{\prime} / 16$, where $r_{0 P}{ }^{\prime}$ is the size of beam in this plane. The error of intensity calculations was assessed by comparing the results with data obtained by processing of the etalon vortex. This error was less than 3\%.

Additional errors appear at rotation of the field, but rotation on angle of $180^{\circ}$ always gives zero error, and on angle $\Delta=120^{\circ}$, the following errors: $0.0003(0.22 \%)$ for $V_{d}=-1,0.0029(0.1 \%)$ for $V_{d}=-2$, and $0.0012(0.12 \%)$ for $V_{d}=-3$. To make numeric experiments more realistic, we introduced in the output plane of the generator relative shifts of receiver and generator optical axes: $S_{h} \equiv\left|\left(S_{h x}, S_{h y}\right)\right|=$ $\left|-0.079 r_{0 B}(1,1)\right|=0.11 r_{0 B}$, which results in shift of axes in the plane of receiver on the value of $S_{h}{ }^{\prime}=0.044 r_{0 B}{ }^{\prime}$. These operations induced increase of errors in calculation of $I_{r}$ up to $3 \%$ for angle of $180^{\circ}$ and up to $10 \%$ for $\Delta=120^{\circ}$. Nevertheless, these errors can be considered as systematic ones, and their influence on precision of vortex registration can be reduced by variations of threshold values in (22).

The obtained dependence of $I_{r}$ on Fried's coherence length is shown in Figure 6 for 3 different topological charges $\left(V_{d}=-1, V_{d}=-2\right.$, and $V_{d}=-3$ ). Under conditions of weak turbulence $\left(L_{F} / r_{0 B} \cong 50\right)$ for $\Delta=120^{\circ}$ and $V_{d}=-3$, the value of $I_{r}$ differs from unity on $1.4 \%$, that is, deviation of $I_{r}$ almost coincides with theoretical assessments (4\%) obtained earlier.

Analysis of results obtained numerically and partially represented in Figure 6 reveals the following features of the detection system.

(1) In weak turbulence $\left(L_{F}>10 r_{0 B}\right)$ intensity, $I_{r}$ does not change. Values of $I_{r}$ are close to corresponding results registered in the presence of weak white noise: $I_{r}=0$ and $I_{r}=2$ in Figure 2 with $\Delta=180^{\circ}$; $I_{r}=0.14$ and $I_{r}=1.86$ in Figure 3 with $\Delta=$ $120^{\circ}$. Differences of registered values of intensity are induced mainly by shifts of optical axes and, to smaller extent, by atmospheric turbulence.

(2) Concurrence of graphics obtained for paths of 5.9 and $59 \mathrm{~km}$ confirms the theoretical statement according to which the value of relative intensity $I_{r}$ does not depend on the path length. 


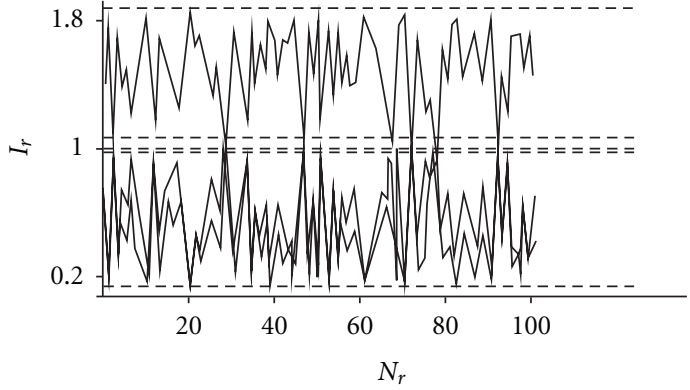

(a)

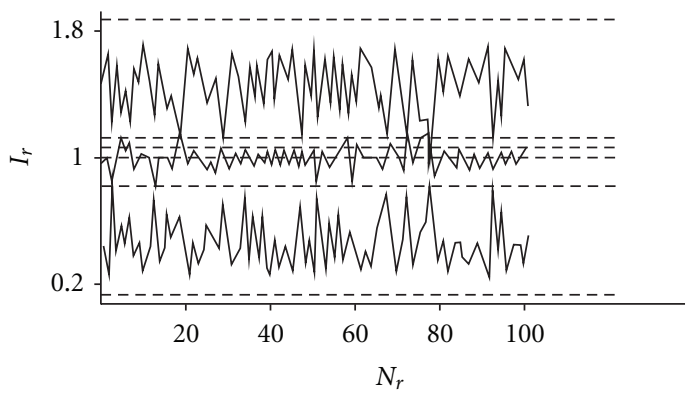

(c)

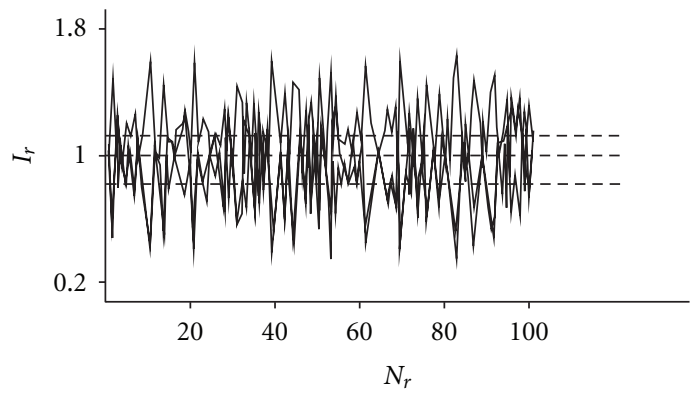

(b)

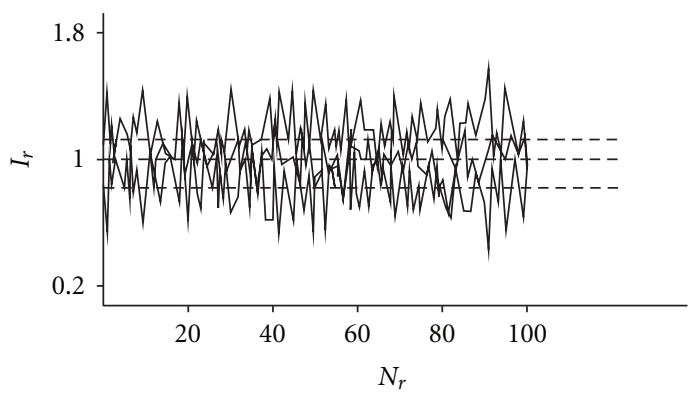

(d)

FIGURE 7: Relative intensity $I_{r}$ calculated numerically with application of different phase screens $N_{r}\left(N_{r} \in[0,99]\right)$. Angles of field rotation $\Delta=180^{\circ}(\mathrm{a}, \mathrm{b})$ and $\Delta=120^{\circ}(\mathrm{c}, \mathrm{d})$, Fried's coherence length $L_{\mathrm{F}}=0.25 r_{0 B}(\mathrm{a}, \mathrm{c})$ and $L_{\mathrm{F}}=0.1 r_{0 B}(\mathrm{~b}, \mathrm{~d})$. The uppermost and bottommost dashed lines correspond to values of $I_{r}=1.86$ and 0.14 . Dashed lines in the central part of the picture correspond to $I_{r}=1$ with threshold values of 0.97 and 1.06 ( $\mathrm{a}$ and $\mathrm{c}$ ) and to $I_{r}=1$ and threshold values of 0.82 and 1.12 (b and d).

(3) Analysis of topological charge detection in a single realization allows us to conclude that

(i) if the angle of field rotation is $180^{\circ}$, vortices with topological charges $V_{d}=-1$ and $V_{d}=-2$ can be detected when $L_{\mathrm{F}}>0.26 r_{0 B}$, with $V_{d}=$ -2 and $V_{d}=-3$ when $L_{\mathrm{F}}>0.26 r_{0 B}$ (the borders of regions are shown in Figure 6(a) by the first and third dashed lines);

(ii) if $\Delta=120^{\circ}$, vortices with $V_{d}=-1$ and $V_{d}=$ -3 can be detected when $L_{\mathrm{F}}>0.71 r_{0 B}$, with $V_{d}=-2$ and $V_{d}=-3$ when $L_{\mathrm{F}}>0.43 r_{0 B}$ (the fourth and fifth dashed lines in Figure 6(b));

(4) To detect vortices with topological charges $V_{d}=-1$ and $V_{d}=-2$ or $V_{d}=-2$ and $V_{d}=-3$ and with rotation of the field on angle $\Delta=180^{\circ}$ we should change the rule of detection given by (22) to the following form:

$$
\begin{aligned}
V_{d} & = \begin{cases}1, & I_{r} \leq 0.97, \\
2, & I_{r}>0.97,\end{cases} \\
\text { or } V_{d} & = \begin{cases}3, & I_{r} \leq 1.063, \\
2, & I_{r}>1.063 .\end{cases}
\end{aligned}
$$

This rule is valid with $L_{F}>0.26 r_{0 B}$ or with $L_{F}>$ $0.26 r_{0 B}$ (corresponding borders are shown by vertical dashed lines in Figure 6(a)).
(5) For angle of rotation $\Delta=120^{\circ}$ in (22) we can change the threshold values and obtain the new condition of vortex detection:

$$
V_{d}= \begin{cases}2+3 i, & I_{r}<0.818 \\ 0+3 i, & I_{r} \in[0.818,1.12], \\ 1+3 i, & I_{r}>1.12\end{cases}
$$

The new rule is valid for detection of vortices with $V_{d}=-1$ and $V_{d}=-3$ or with $V_{d}=-2$ and $V_{d}=-3$ when $L_{F}>0.25 r_{0 B}$ or $L_{F}>0.30 r_{0 B}$ (the regions are marked by the second and third vertical dashed lines in Figure 6(b)). But if we need to detect vortices only with charged $V_{d}=-1$ and $V_{d}=-2$, then we can use the rule

$$
V_{d}= \begin{cases}1, & I_{r} \leq 0.904, \\ 2, & I_{r}>0.904,\end{cases}
$$

which is valid for $L_{\mathrm{F}}>0.216 r_{O B}$ (the first vertical dashed line in Figure 6(b)).

(6) Analysis of large samplings (100 realizations or more) allows us to detect topological charges of vortices with (22) for $L_{F}=0.1 r_{0 B}$ or even in stronger turbulence. Naturally, in procedure of detection we employ averaged values of intensity $\left\langle I_{r}\right\rangle$ instead of $I_{r}$.

Influence of phase screen simulating turbulent distortions of radiation on detection of vortex topological charges 

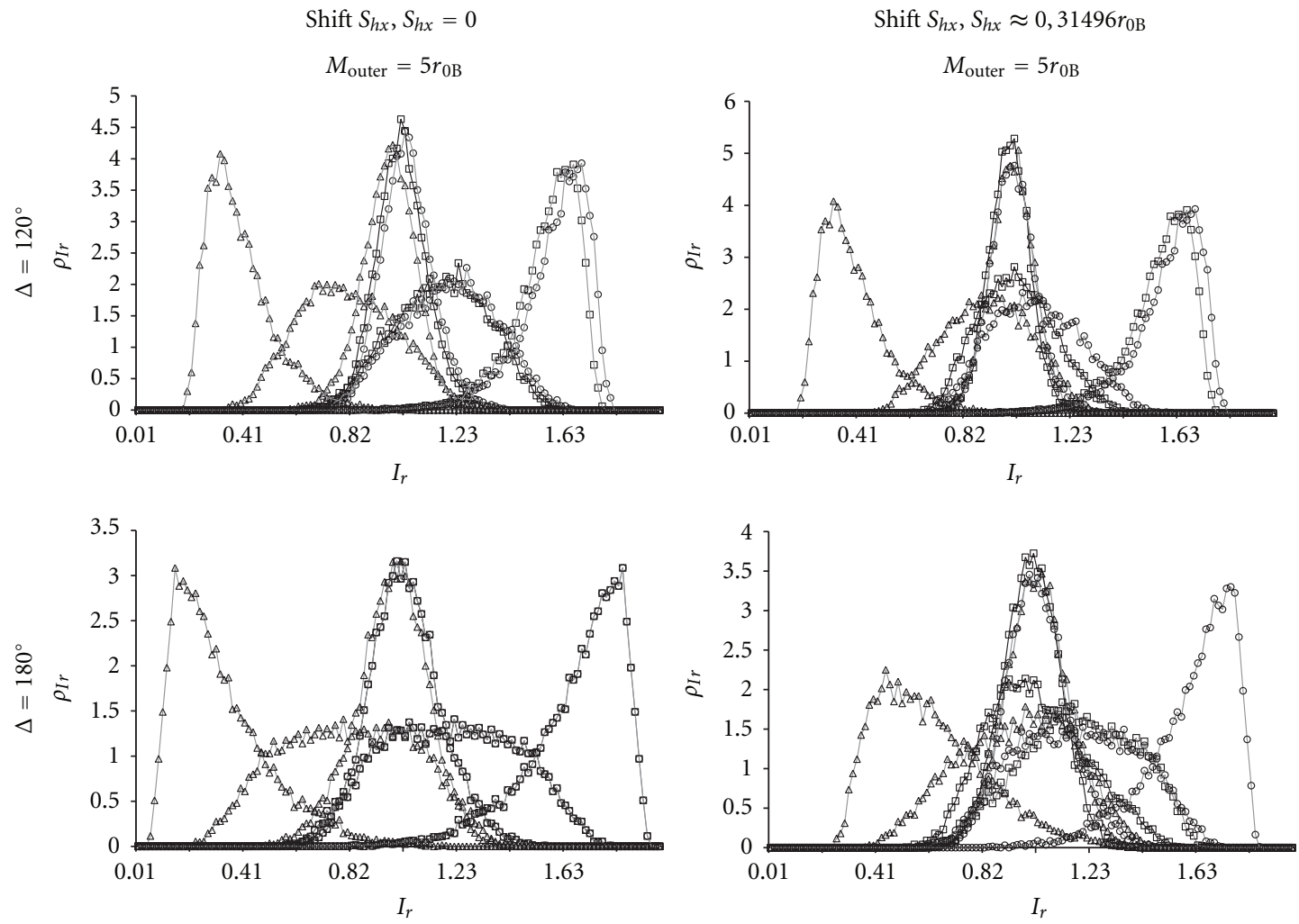

(a)
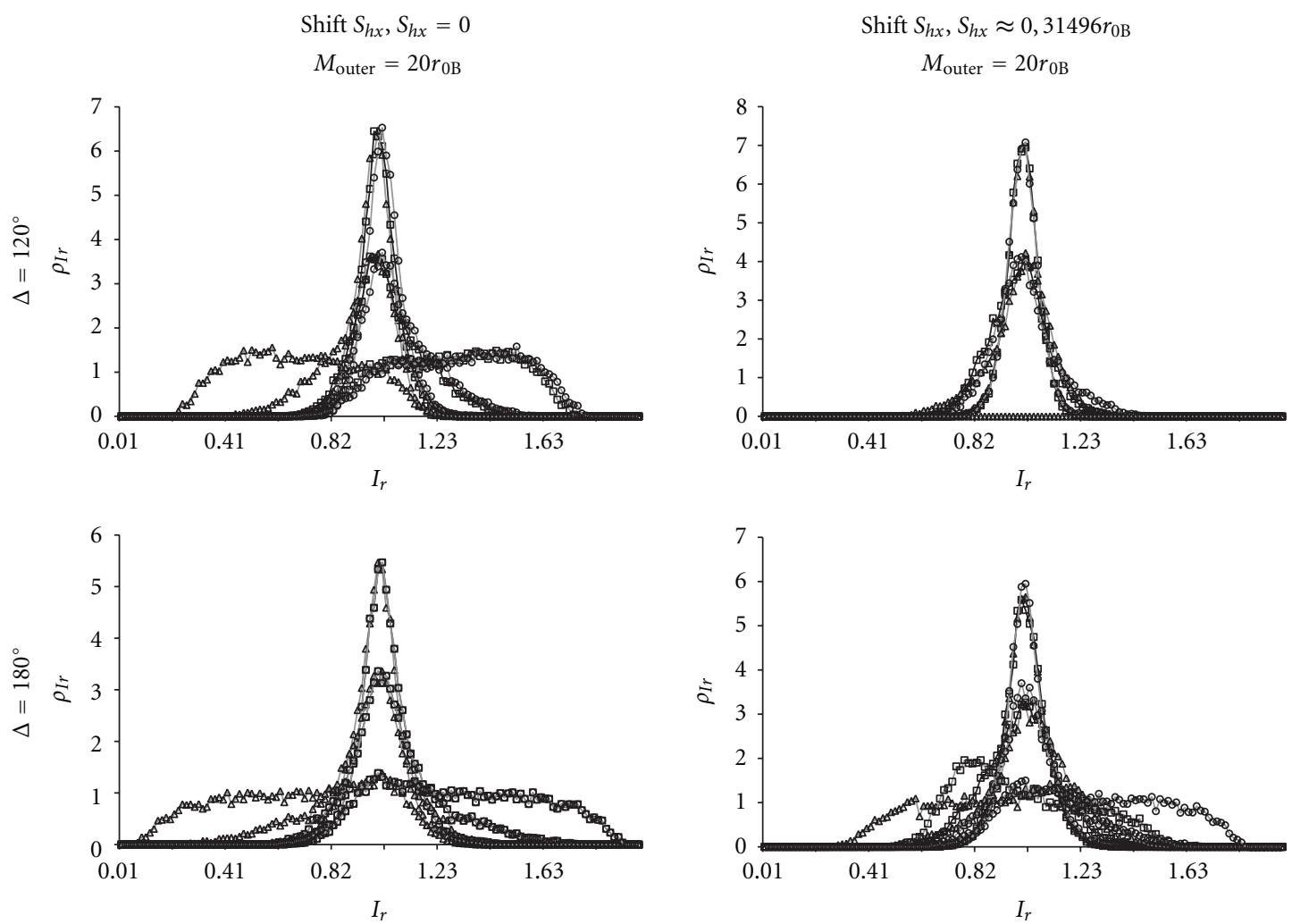

(b)

Figure 8: One-dimensional distributions of probability density $\rho_{I r} \equiv \rho\left(I_{r}\right)$ obtained with different values of $M_{\text {outer }}$ and $S_{h x}$. The values of Fried's coherence length are $0.25,0.1$, and 0.05 . Curves marked by symbols " $\Delta$ ", “ $\square$ ", and "o" were obtained for topological charges $-1,-2$, and 0 correspondingly. 

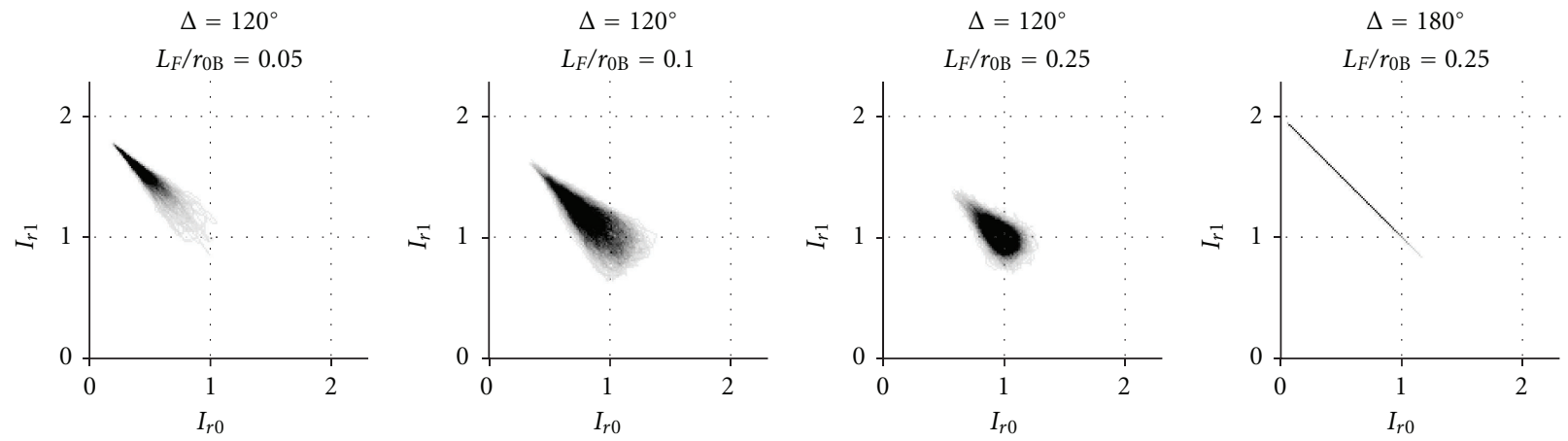

Figure 9: Two-dimensional probability density $\rho\left(I_{r 0}, I_{r 1}\right)$ obtained with $\left(V_{d 0}, V_{d 1}\right)=(-1,-2), S_{h x}=0, M_{\text {outer }}=5 r_{0 B}$, and different values of $L_{F}$ and $\Delta$.
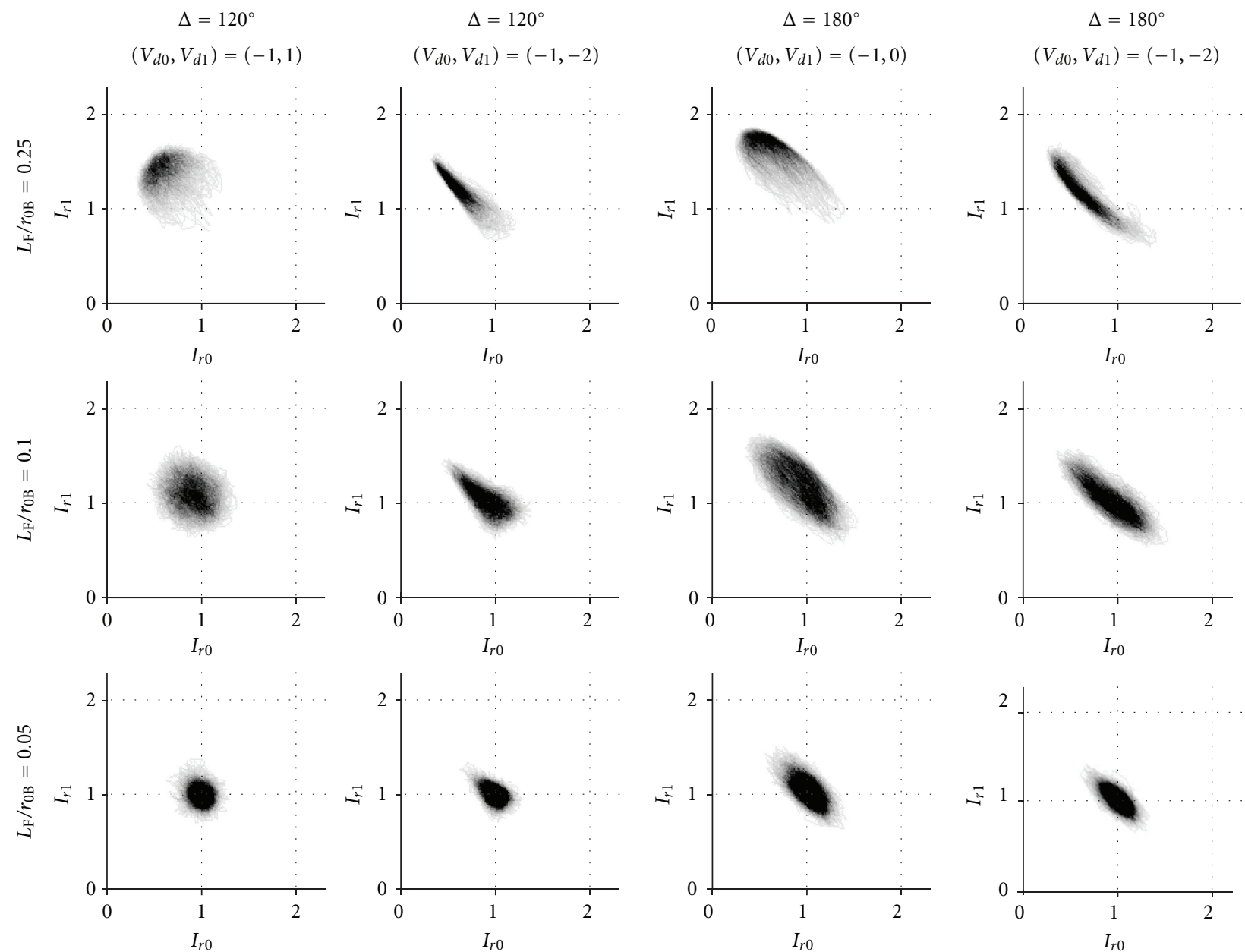

FIGURE 10: Two-dimensional probability density $\rho\left(I_{r 0}, I_{r 1}\right)$ obtained with $S_{h x} \approx 0.314 r_{0 B}, M_{\text {outer }}=5 r_{0 B}$, and different values of $V_{d 0}, V_{d 1}, L_{F}$, and $\Delta$.

is illustrated in Figure 7. Different curves in this picture correspond to vortices with different charges $\left(V_{d}=-2, V_{d}=\right.$ -1 , and $V_{d}=-3$ ). In Figures $7(\mathrm{~b})$ and $7(\mathrm{~d})$, curves cannot be separated which indicates large strength of turbulence.

Also in Figure 7 we observe mirror symmetry between curves $I_{r}\left(N_{r}\right)$ related with different charges, for example, with $V_{d}=-2$ and $V_{d}=-1 ; V_{d}=-2$ and $V_{d}=-3$.
The exact reflection of curves is possible to expect when optical axis of receiver and detector coincide and angle of rotation is $180^{\circ}$, but development of symmetry for $\Delta=120^{\circ}$ is unachievable (Figures 7(c) and 7(d)).

One more characteristic feature observed in these pictures is the following: normally, $I_{r}\left(N_{r}, V_{d 0}\right) \neq I_{r}\left(N_{r}, V_{d 1}\right)$ for $V_{d 0} \neq V_{d 1}$. This attribute and the symmetry of curves are also 

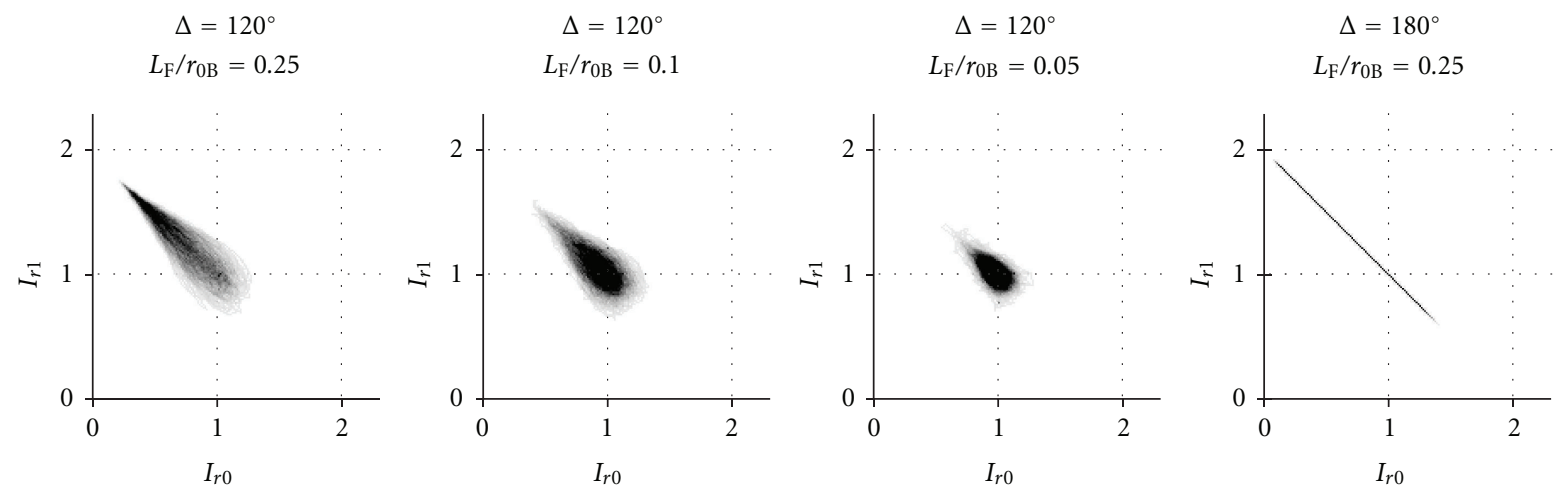

Figure 11: The same data as in Figure 8 but obtained with $M_{\text {outer }}=20 r_{0 B}$.
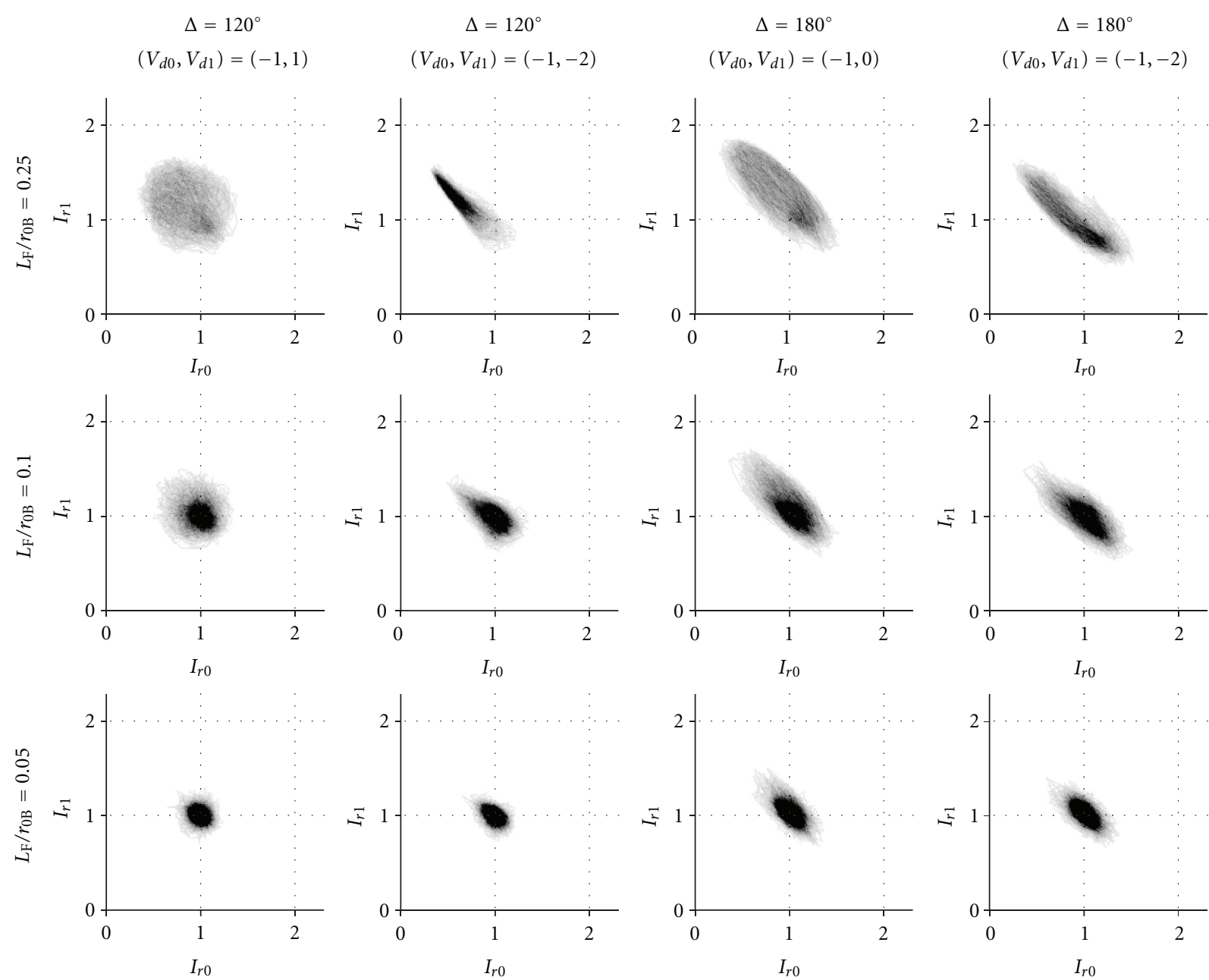

FIGURE 12: The same data as in Figure 9 but obtained with $M_{\text {outer }}=20 r_{0 B}$.

illustrated by distributions $\rho\left(I_{r}\right), \rho\left(I_{r 0}, I_{r 1}\right)$ of probability density presented in Figures 8, 9, 10, and 11 for different values of $L_{\mathrm{F}}, M_{\text {outer }}, S_{h x}$, and $V_{d}$.

Graphs representing the results of numeric experiments with zero shifts of optical axes show that differences in probability density $\rho\left(I_{r 0}, I_{r 1}\right)$ for triplets $\left(120^{\circ},-1,1\right)$ and $\left(120^{\circ},-1,-2\right)$ cannot be distinguished visually. The same is true for triplets $\left(180^{\circ},-1,0\right)$ and $\left(180^{\circ},-1,-2\right)$.

According to Figures 9, 10, 11, and 12 with increase of turbulence strength (with decrease of $L_{\mathrm{F}}$ ), distribution of probability density initially localized in vicinity of point $(1,1)$ moves out of the second quadrant in direction of 
TABLE 1: Averaged over 13000 realizations mean values of $\left\langle I_{r 1}-I_{r 0}\right\rangle$ and maximum amplitudes $I_{r 0 \max }-I_{r 1 \text { min }}$ obtained for pairs ( $V_{d 0}$, $V_{d 1}$ ) with different Fried's coherence lengths $L_{\mathrm{F}} / r_{0 B}$, angles of field rotation $\Delta$, shifts of optical axes $S_{h x}$, and different outer scales $M_{\text {outer }}$.

\begin{tabular}{|c|c|c|c|c|c|c|c|c|c|}
\hline \multirow{4}{*}{$L_{\mathrm{F}} / r_{0 B}$} & \multirow{4}{*}{$\begin{array}{l}V_{d 0} \\
V_{d 1}\end{array}$} & \multicolumn{4}{|c|}{$\Delta=180^{\circ}$} & \multicolumn{4}{|c|}{$\Delta=120^{\circ}$} \\
\hline & & \multicolumn{2}{|c|}{$S_{h x}=0$} & \multicolumn{2}{|c|}{$S_{h x} \approx 0.314 r_{0 B}$} & \multicolumn{2}{|c|}{$S_{h x}=0$} & \multicolumn{2}{|c|}{$S_{h x} \approx 0.314 r_{0 B}$} \\
\hline & & -1 & -1 & -1 & -1 & -1 & -1 & -1 & -1 \\
\hline & & 0 & -2 & 0 & -2 & -2 & +1 & -2 & +1 \\
\hline & & \multicolumn{8}{|c|}{$M_{\text {outer }}=5 r_{0 B}$} \\
\hline \multirow{2}{*}{0.25} & $\left\langle I_{r 1}-I_{r 0}\right\rangle$ & & & 0.997 & 0.56 & 1.16 & 1.20 & 0.60 & 0.79 \\
\hline & $I_{r 0 \max }-I_{r 1 \min }$ & & & 0.57 & 0.83 & 0.20 & 0.18 & 0.55 & 0.45 \\
\hline \multirow{2}{*}{0.10} & $\left\langle I_{r 1}-I_{r 0}\right\rangle$ & & & 0.24 & 0.08 & 0.35 & 0.38 & 0.11 & 0.19 \\
\hline & $I_{r 0 \max }-I_{r 1 \min }$ & & & 1.00 & 1.02 & 0.75 & 0.72 & 0.80 & 0.76 \\
\hline \multirow{2}{*}{0.05} & $\left\langle I_{r 1}-I_{r 0}\right\rangle$ & & & 0.01 & -0.02 & 0.05 & 0.07 & -0.01 & 0.01 \\
\hline & $I_{r 0 \max }-I_{r 1 \min }$ & & & 0.79 & 0.80 & 0.59 & 0.57 & 0.60 & 0.59 \\
\hline & & \multicolumn{8}{|c|}{$M_{\text {outer }}=20 r_{0 B}$} \\
\hline \multirow{2}{*}{0.25} & $\left\langle I_{r 1}-I_{r 0}\right\rangle$ & & & 0.36 & 0.08 & 0.58 & 0.62 & 0.19 & 0.32 \\
\hline & $I_{r 0 \max }-I_{r 1 \min }$ & & & 0.91 & 1.03 & 0.61 & 0.59 & 0.82 & 0.78 \\
\hline \multirow{2}{*}{0.10} & $\left\langle I_{r 1}-I_{r 0}\right\rangle$ & & & 0.03 & -0.03 & 0.12 & 0.13 & -0.01 & 0.02 \\
\hline & $I_{r 0 \max }-I_{r 1 \min }$ & & & 0.98 & 1.02 & 0.72 & 0.71 & 0.74 & 0.71 \\
\hline \multirow{2}{*}{0.05} & $\left\langle I_{r 1}-I_{r 0}\right\rangle$ & & & 0.01 & -0.00 & 0.02 & 0.04 & -0.00 & 0.00 \\
\hline & $I_{r 0 \max }-I_{r 1 \min }$ & & & 0.72 & 0.73 & 0.60 & 0.57 & 0.51 & 0.54 \\
\hline
\end{tabular}

TABLE 2: Influence of optical axes shift $S_{h x}$ and outer scale $M_{\text {outer }}$ of turbulence on difference $I_{r 0 \max }-I_{r 1 \text { min }}$ and mean difference $\left\langle I_{r 1}-I_{r 0}\right\rangle$ of functions $I_{r 1}\left(N_{r}\right)$ and $I_{r 0}\left(N_{r}\right)$.

\begin{tabular}{|c|c|c|c|c|c|c|c|c|}
\hline \multirow{4}{*}{ Difference } & \multicolumn{4}{|c|}{ Shift of axes $S_{h x} / r_{0 B}$} & \multicolumn{4}{|c|}{ Outer scale of turbulence $M_{\text {outer }} / r_{0 B}$} \\
\hline & \multirow{3}{*}{0} & \multicolumn{3}{|c|}{ Increase: $0 \rightarrow 0.315$} & \multirow{3}{*}{5} & \multirow{3}{*}{20} & \multirow{2}{*}{\multicolumn{2}{|c|}{$\begin{array}{c}\text { Increase: } 5 \rightarrow 20 \\
\text { Variations, \% }\end{array}$}} \\
\hline & & 0.315 & Varia & $\%$ & & & & \\
\hline & & & Interval & Mean & & & Interval & Mean \\
\hline$\left\langle I_{r 1}-I_{r 0}\right\rangle$ & 0.41 & $\begin{array}{c}0.188 \approx \\
\approx(1-0.54) \cdot 0.41\end{array}$ & $\begin{array}{c}-131 \cdots \\
-26\end{array}$ & -77 & 0.432 & $\begin{array}{c}0.164 \approx \\
\approx(1-0.62) \cdot 0.432\end{array}$ & $\begin{array}{c}-144 \cdots \\
-31.7\end{array}$ & -70 \\
\hline$I_{r 0 \max }-I_{r 1 \min }$ & 0.68 & $\begin{array}{c}0.76 \approx \\
\approx(1+0.11) \cdot 0.68\end{array}$ & $\begin{array}{c}-17 \cdots \\
63\end{array}$ & 12 & 0.77 & $\begin{array}{c}0.67 \approx \\
\approx(1-0.14) \cdot 0.77\end{array}$ & $\begin{array}{c}-229 \cdots \\
+15\end{array}$ & -34 \\
\hline
\end{tabular}

the fourth one and acquires symmetric shape relatively to this point. As a result distribution of function $\rho\left(I_{r 0}, I_{r 1}\right)$ for $L_{\mathrm{F}} / r_{0 P}=0.05$ looks like a distribution of the Gaussian function.

Relative shift of the generator and receiver optical axes $S_{h x} \approx 0.314 r_{0 B}$ in the presence of weak turbulence $\left(L_{\mathrm{F}}=\right.$ $0.25 r_{0 P}$ ) results in the broadening of distribution and transport of its gravity center to the first and third quadrants. These changes are clearly seen in graphics corresponding to $\Delta=180^{\circ}$. With increase of turbulence, intensity increases the speed of transformation of $\rho\left(I_{r 0}, I_{r 1}\right)$ distribution to a Gaussian form.

Symmetry of $\rho\left(I_{r 0}, I_{r 1}\right)$ distribution guarantees correct data transfer by a system even on realizations when the topological charge of vortex is incorrectly detected. Due to symmetry an error of vortex identification is possible to consider as a regular event; therefore, it can be taken into account in the algorithm of detection. Another property of the system, namely, the difference of intensities $I_{r}\left(N_{r}, V_{d 0}\right) \neq I_{r}\left(N_{r}, V_{d 1}\right)$ observed when $V_{d 0} \neq V_{d 1}$ suggests application of adaptive thresholds in the algorithm, with the use of which the errors can be reduced.
The above-presented conclusions based on visual analysis of results are supported by the data of Table 1, where the influence of phase screen, detector, and order of a singular points on difference $\left\langle I_{r}\left(N_{r}, V_{d 1}\right)-I_{r}\left(N_{r}, V_{d 0}\right)\right\rangle_{N_{r}} \equiv\left\langle I_{r 1}-\right.$ $\left.I_{r 0}\right\rangle$ is illustrated. Obviously, identification of vortices is easier if the difference of intensities $\left\langle I_{r 1}-I_{r 0}\right\rangle$ is greater. According to Table 1 detection of vortices is not always possible even with small intensity of turbulence, for example, with $L_{F}=0.25 r_{0 B}$. This notion does not conflict with conclusions 3-5 drawn for $S_{h}=0.11 r_{0 B}$ and $M_{\text {outer }}=5 r_{0 P B}$ in contrast with the thesis about possible application of (25) for $L_{\mathrm{F}}>0.216 r_{0 P}$ which seems rather doubtful. The cause of disagreement can be explained as follows: according to our estimates, probability of the event appearance is rather small (about 1.6\%) for a random phase screen with $L_{\mathrm{F}}=$ $0.25 r_{0 B}$ and $M_{\text {outer }}=5 r_{0 B}$ which guarantees fulfillment of inequality $I_{r}\left(V_{d 1}=-2\right)<I_{r}\left(V_{d 0}=-1\right)$. But we used only 100 realizations to obtain data shown as graphs in Figure 6, so it is possible to expect that the screen is absent in this sampling, while results in Table 1 were averaged over 13000 realizations. 
The results of Table 1 also allow one to conclude that with $\Delta=180^{\circ}$ and $S_{h x}=0$, values of $\left\langle I_{r 1}-I_{r 0}\right\rangle$ and $I_{r 0 \max }-I_{r 1 \min }$ obtained for different pairs of $\left(V_{d 0}, V_{d 1}\right)$ coincide precisely. This property can be attributed to mirror symmetry of corresponding curves representing functions $I_{r}\left(N_{r}\right)$. With $\Delta=120^{\circ}$ and $S_{h x}=0$, between values of $\left\langle I_{r 1}-I_{r 0}\right\rangle$ and $I_{r 0 \max }-I_{r 1 \mathrm{~min}}$ we registered only small difference. If the optical axes are shifted, mirror symmetry of curves is broken as well as correlation between values of $\left\langle I_{r 1}-I_{r 0}\right\rangle$ and $I_{r 0 \max }-I_{r 1 \mathrm{~min}}$.

Additionally, increase of $S_{h x}$ and of the outer scale $M_{\text {outer }}$ results in decrease of $\left\langle I_{r 1}-I_{r 0}\right\rangle$ (Table 2). Obviously, in this case, performance of the detector can be improved with application of an adaptive optics system compensating for large-scale aberrations of radiation.

\section{Conclusions}

The main results of theoretical analysis can be summarized as follows.

(1) Due to optical field transformation in an interferometer, the magnitude of the output intensity depends on the order of a vortex carrying by the field. This magnitude can be used to detect presence and order $V_{d}$ of the vortex.

(2) The influence of white amplitude and phase noise was assessed on precision of vortex identification. It was shown that precision of vortex identification is influenced mainly by amplitude white noise.

(3) Invariance of detector characteristics under path length was proved in linear homogeneous medium.

(4) The mirror symmetry of the output field intensity $I_{r}$ relatively to the level $I_{r}=1$ for specific angles of rotation, and specific differences of topological charges was demonstrated.

With the use of numerical simulation, it was shown that

(1) Assessments of white noise and turbulence influence on precision of a vortex detection support theoretical results.

(2) The simple criteria are possible to introduce in the model of a detector to distinguish presence and order of optical vortex.

(3) Magnitude of relative intensity $I_{r}$ and precision of vortex detection depend noticeably on shifts of receiver and source optical axes.

The main characteristics are also found of $1 \mathrm{D}$ and $2 \mathrm{D}$ probabilities of vortex detection as functions of Fried's coherence length, inner and outer scales of turbulence, and relative shifts of optical axes.

\section{References}

[1] L. Allen, M. W. Beijersbergen, R. J. C. Spreeuw, and J. P. Woerdman, "Orbital angular momentum of light and the transformation of Laguerre-Gaussian laser modes," Physical Review A, vol. 45, no. 11, pp. 8185-8189, 1992.
[2] G. Molina-Terriza, J. P. Torres, and L. Torner, "Management of the angular momentum of light: preparation of photons in multidimensional vector states of angular momentum," Physical Review Letters, vol. 88, no. 1, pp. 136011-136014, 2002.

[3] G. Gibson, J. Courtial, M. J. Padgett et al., "Free-space information transfer using light beams carrying orbital angular momentum," Optics Express, vol. 12, no. 22, pp. 5448-5456, 2004.

[4] C. Paterson, "Atmospheric turbulence and orbital angular momentum of single photons for optical communication," Physical Review Letters, vol. 94, no. 15, Article ID 153901, 2005.

[5] V. Aksenov, I. Izmailov, F. Kanev, and B. Poizner, "Detector of topological charges of optical vortices," in Proceedings of the International Conference "Laser Optics '10", p. 21, St.Petersburg, Russia, 2010.

[6] R. B. Shack and B. C. Platt, "Production and use of a lenticular hartmann screen," Journal of the Optical Society of America, vol. 61, pp. 656-662, 1971.

[7] F. A. Starikov, V. P. Aksenov, F. Y. Kanev et al., "Wavefront reconstruction of an optical vortex by a Hartmann-Shack sensor," Optics Letters, vol. 32, no. 16, pp. 2291-2294, 2007.

[8] V. Voitsekhovich, L. Sanchez, V. Orlov, and S. Cuevas, "Efficiency of the Hartmann test with different subpupil forms for the measurement of turbulence-induced phase distortions," Applied Optics, vol. 40, no. 9, pp. 1299-1304, 2001.

[9] R. Ragazzoni, "Pupil plane wavefront sensing with an oscillating prism," Journal of Modern Optics, vol. 43, no. 2, pp. 289293, 1996.

[10] R. Ragazzoni, A. Ghedina, A. Baruffolo et al., "Testing the pyramid wavefront sensor on the sky," in The International Society for Optics and Photonics, vol. 4007 of Proceedings of the SPIE, pp. 423-429, March 2000.

[11] M. Ghigo, G. Crimi, and F. Perennes, "Construction of a pyramidal wavefront sensor for adaptive optics compensation," in Proceedings of the International Conference "Beyond Conventional Adaptive Optics", pp. 465-472, 2001.

[12] F. Y. Kanev, V. P. Lukin, and N. A. Makenova, "Detection of dislocations as branching points of interference pattern," in The International Society for Optics and Photonics, vol. 4357 of Proceedings of the SPIE, pp. 231-235, June 2001.

[13] C. Rockstuhl, A. A. Ivanovskyy, M. S. Soskin, M. G. Salt, H. P. Herzig, and R. Dändliker, "High-resolution measurement of phase singularities produced by computer-generated holograms," Optics Communications, vol. 242, no. 1-3, pp. 163169, 2004.

[14] M. V. Berry, "Coloured phase singularities," New Journal of Physics, vol. 4, pp. 66.1-66.14, 2002.

[15] M. V. Berry, "Exploring the colours of dark light," New Journal of Physics, vol. 4, pp. 74.1-74.14, 2002.

[16] G. V. Bogatyryova, C. V. Felde, P. V. Polyanskii, S. A. Ponomarenko, M. S. Soskin, and E. Wolf, "Partially coherent vortex beams with a separable phase," Optics Letters, vol. 28, no. 11 , pp. 878-880, 2003.

[17] D. L. Fried, "Adaptive optics wave function reconstruction and phase unwrapping when branch points are present," Optics Communications, vol. 200, no. 1-6, pp. 43-72, 2001.

[18] V. P. Aksenov, I. V. Izmailov, F. Y. Kanev, and F. A. Starikov, "Algorithms for the reconstruction of the singular wave front of laser radiation: analysis and improvement of accuracy," Quantum Electronics, vol. 38, no. 7, pp. 673-677, 2008.

[19] I. V. Izmailov, A. L. Magazinnikov, and B. N. Poizner, "Identification of a screw wave-front dislocation and compensation for its influence on structurization in models of a ring interferometer," Atmospheric and Ocean Optics, vol. 13, pp. 805-812, 2000. 

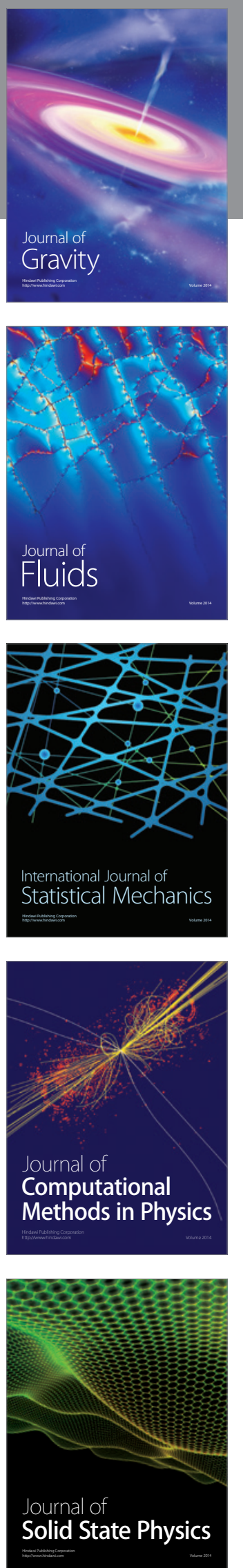

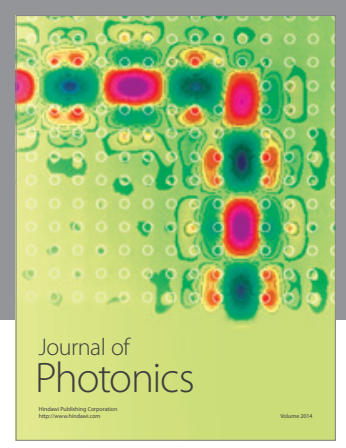

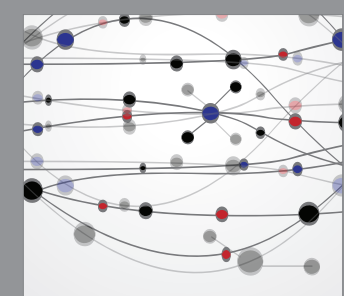

The Scientific World Journal
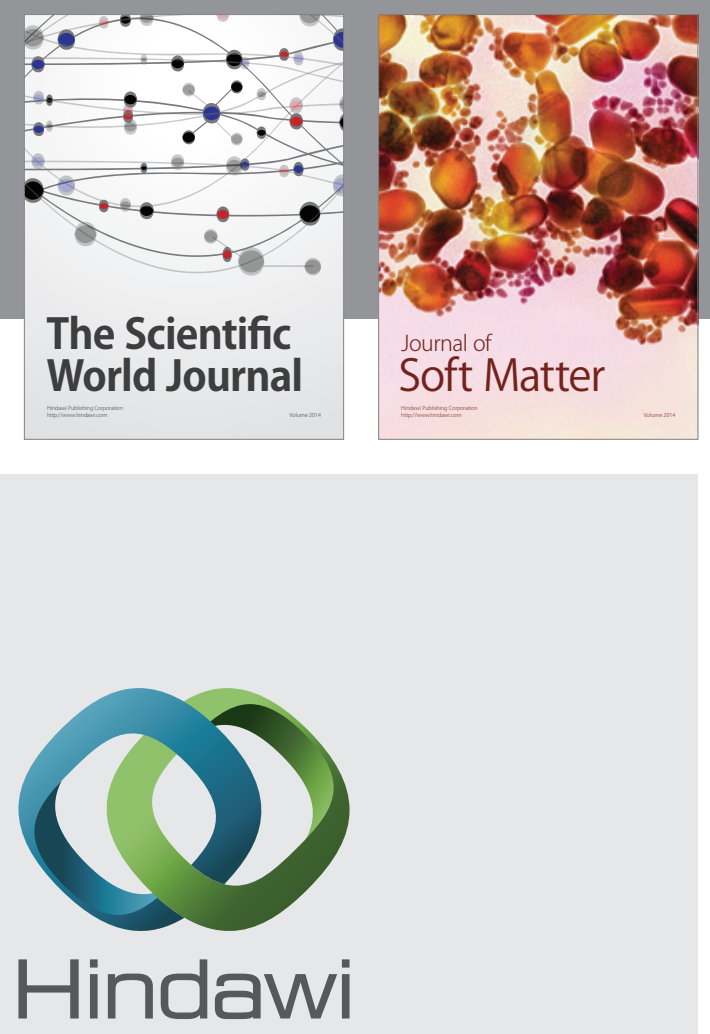

Submit your manuscripts at

http://www.hindawi.com
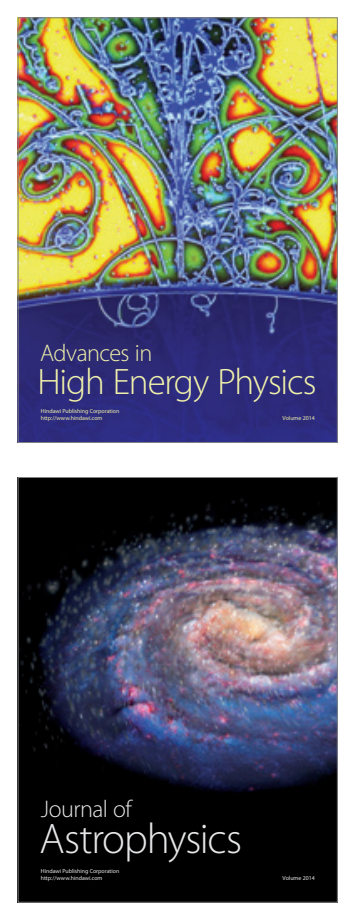
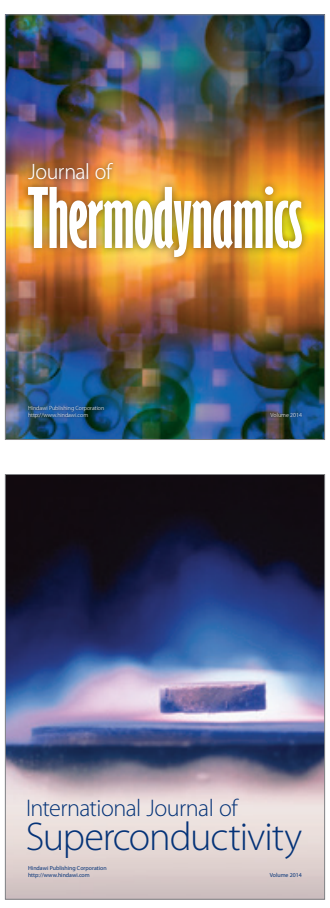
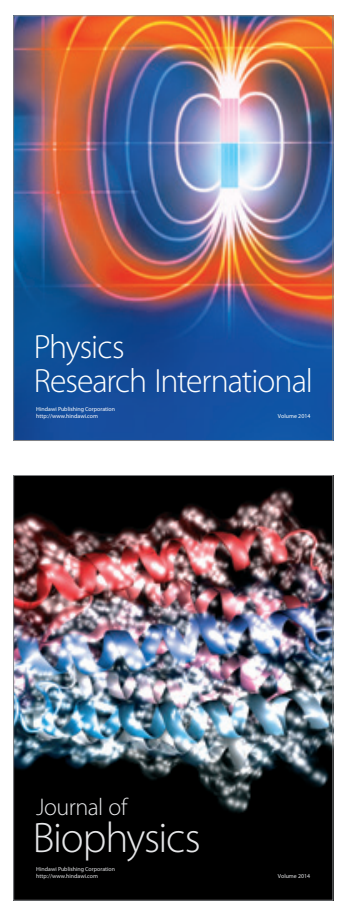
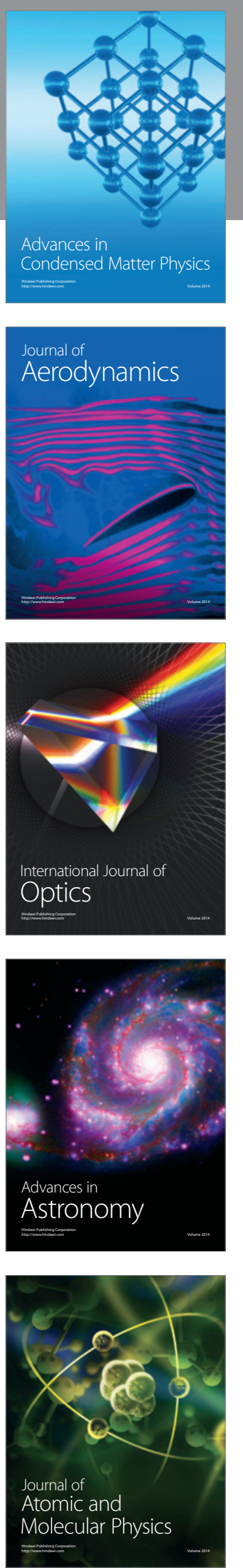